INDOOR LOCATION TRACKING AND ORIENTATION ESTIMATION USING A PARTICLE FILTER, INS, AND RSSI

\author{
A Thesis \\ presented to \\ the Faculty of California Polytechnic State University, \\ San Luis Obispo
}

\author{
In Partial Fulfillment \\ of the Requirements for the Degree \\ Master of Science in Agriculture
}

by

Cameron Nouri

December, 2014 
(c) 2014

Cameron Nouri

Page i 
ALL RIGHTS RESERVED

COMMITTEE MEMBERSHIP

TITLE:

Indoor Location Tracking and Orientation Estimation using a Particle Filter, INS, and RSSI

AUTHOR:

Cameron Nouri

DATE SUBMITTED:

December, 2014

COMMITTEE CHAIR:

Dr. Tina Smilkstein, Assistant Professor

Electrical Engineering Department

COMMITTEE MEMBER:

Dr. Bridget Benson, Assistant Professor Electrical Engineering Department

COMMITTEE MEMBER:

Dr. Zoe Wood, Associate Professor

Computer Science Department 


\section{ABSTRACT \\ Indoor Location Tracking and Orientation Estimation using a Particle Filter, INS, and RSSI Cameron Nouri}

With the advent of wireless sensor technologies becoming more and more common-place in wearable devices and smartphones, indoor localization is becoming a heavily researched topic. One such application for this topic is in the medical field where wireless sensor devices that are capable of monitoring patient vitals and giving accurate location estimations allow for a less intrusive environment for nursing home patients.

This project explores the usage of using received signal strength indication (RSSI) in conjunction with an inertial navigation system (INS) to provide location estimations without the use of GPS in a Particle Filter with a small development microcontroller and base station. The paper goes over the topics used in this thesis and the results. 


\section{ACKNOWLEDGMENTS}

Thank you Rudi Bendig and Ryan Green and Kenneth Finnigan for information at the onset of this thesis. Thank you Collin MacGregor and Karl Preheim for the help in reviewing data and providing input. Thank you to my advisor committee for the knowledge and helpful tutelage throughout my college career. 
LIST OF TABLES ..........................................................................................................

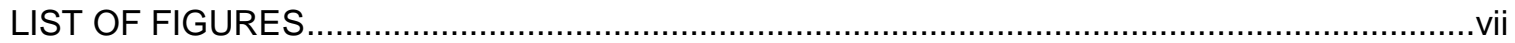

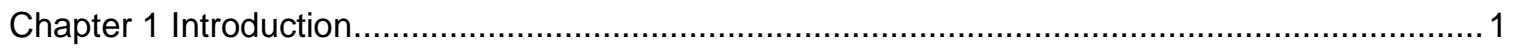

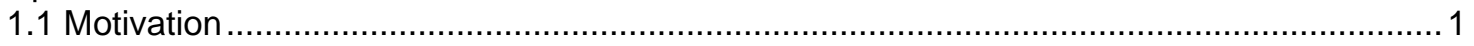

1.2 Background and Related Works ...........................................................................

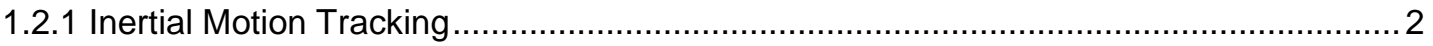

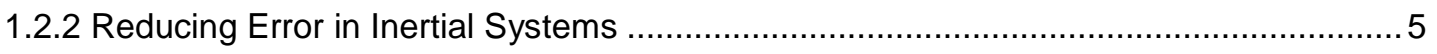

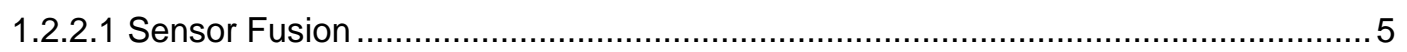

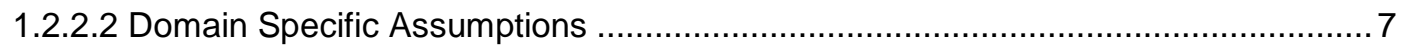

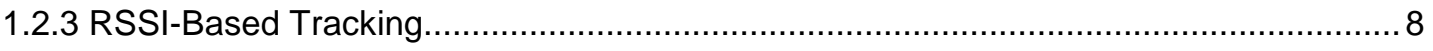

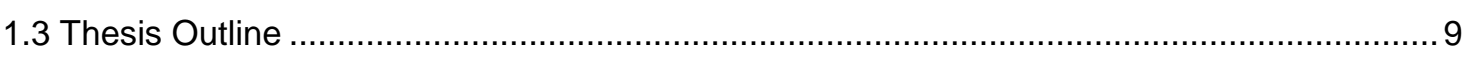

Chapter 2 System Model..............................................................................................

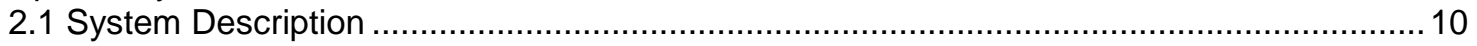

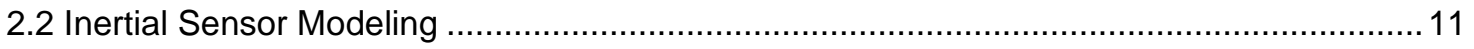

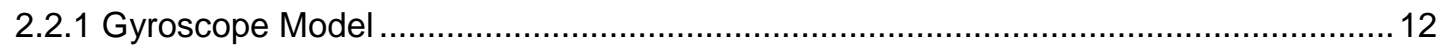

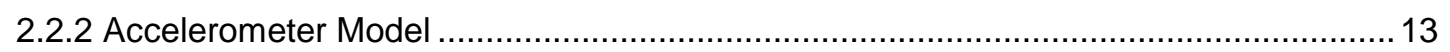

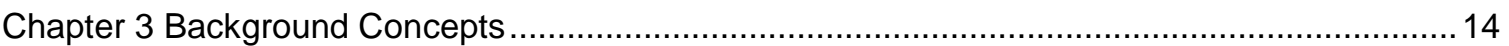

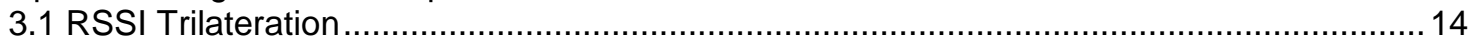

3.1.1 RSS Ranging in Indoor Environment ............................................................... 16

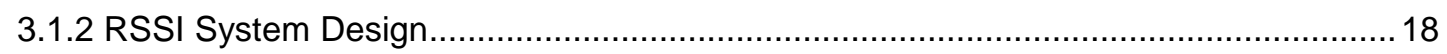

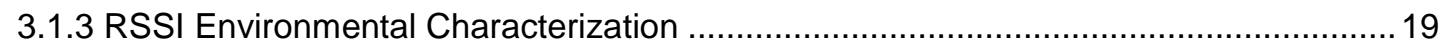

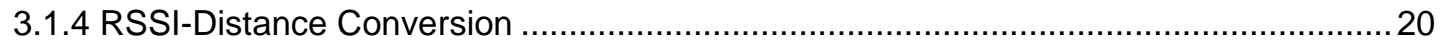

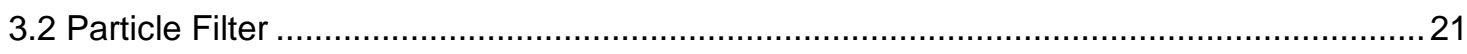

3.2.1 Particle Filter Nomenclature...............................................................................21

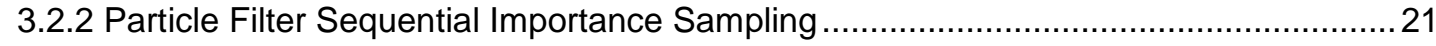

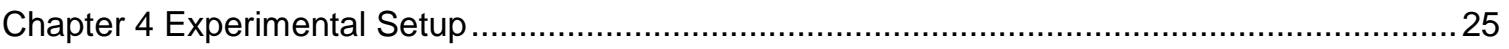

4.1 System Setup

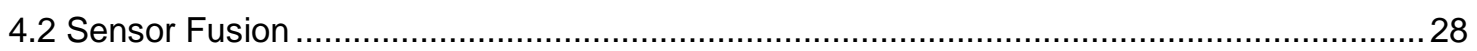

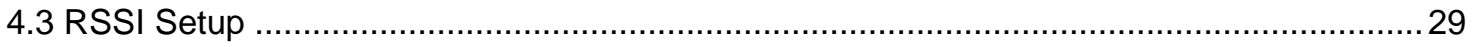

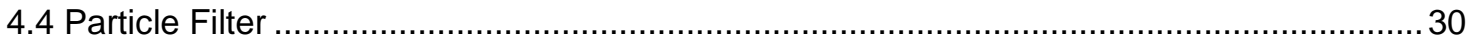

4.4.1 Particle Filter Characterization ...........................................................................

Chapter 5 Results and Analysis ....................................................................................

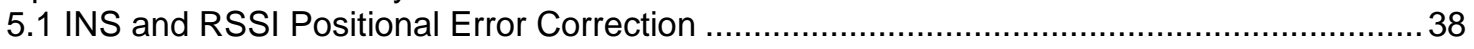

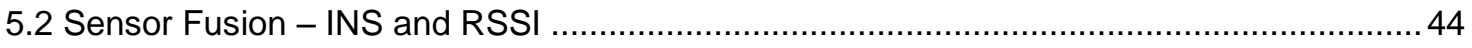

Chapter 6 Discussion and Future Works ........................................................................... 49

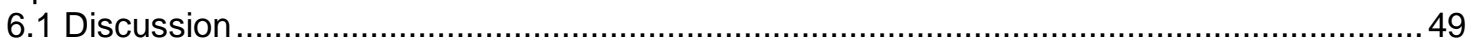

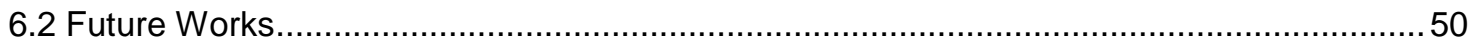

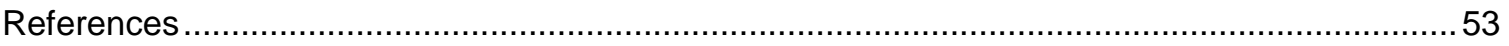




\section{LIST OF TABLES}

\section{Page}

Table 1: Environmental characterization step between stationary nodes and mobile nodes

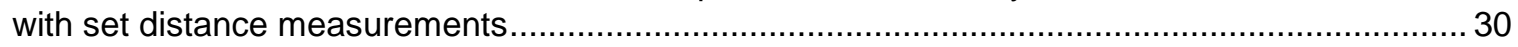

Page vi 


\section{LIST OF FIGURES}

Figure

Figure 1: Comparison of 1- $\sigma$ random position drift performance of commercial, tactical, navigation, strategic-grade, and "perfect" inertial navigation systems over a 20 minute covariance simulation [8].

Figure 2: Example of gyroscope drift over time. Blue line indicates drift over time, red indicates the value of the gyroscope. [29].

Figure 3: [10] System description diagram. The diagram shows the mobile node, the anchor nodes, and the control station (or base station) and the relationship between them. The anchor nodes send packets to the mobile node, and the mobile node sends all resulting information from the gyroscope, accelerometer, and RSSI information to the control station. The control station aggregates, and estimates location utilizing both the inertial measurements and RSSI-based estimates.

Figure 4: The sensor model diagram and its relations. The diagram details the inputs and outputs of the sensors used on the mobile node and their interaction between each other. Gaussian white noise is added to each of the signals, and a slight biasing signal is applied to the gyroscope upon startup. The orientation is a combination of the accelerometer and gyroscope to correct for gyroscope drift.

Figure 5: Trilateration Estimation [26] which shows the concentric circles used by the RSSI ranging technique to calculate the position using $R_{1}, R_{2}, R_{3}$. The mobile node, $T_{1}$, is at the center-point of all three circles of each anchor node.

Figure 6: Inverse Square Law, general. [30]

Figure 7: Effects of path exponent [24]

Figure 8: The Findings of Location from Raw RSSI [26]

Figure 9: Overall System Block Diagram [26]

Figure 10: Waspmote with XBEE 802.15.4 PRO module.

Figure 11: Block diagram of the experimental flow of the sensor hardware, both stationary and mobile and how they interact. Location estimation is not done on the hardware, but instead done at the base station. The sync timing block is used to make sure that information transmitted does not overpower other nodes. This allows for a more consistent delay between packets. 
Figure 12: Block diagram of the experimental flow of the base station and the actions the base stations do.

Figure 13: ITG-3200 breakout board [33].

Figure 14: The layout of the $3 \mathrm{~m} \times 4 \mathrm{~m}$ room. Nodes located at the corners.

Figure 15: Measurement Noise with mean of 0 and sigma of 1, System Noise with mean of 0 and sigma of 1

Figure 16: Measurement Noise with mean of 0 and sigma of 5, System Noise with mean of 0 and sigma of 1

Figure 17: Measurement Noise with mean of 0 and sigma of 10, System Noise with mean of 0 and sigma of 1

Figure 18: System Noise with mean 0 and sigma of 5, Measurement Noise with mean 0 and sigma of 1

Figure 19: System Noise with mean 0 and sigma of 10, Measurement Noise with mean 0 and sigma of 1

Figure 20: Stationary Poor INS Starting condition with actual node position at $(1.5 \mathrm{~m}, 2.0 \mathrm{~m})$

Figure 21: Corrected Stationary Poor INS Starting condition with actual node position at $(1.5 \mathrm{~m}, 2.0 \mathrm{~m})$.

Figure 22: Vertical movement, Y-axis only. Gyroscopic drift in X direction. Without INS positional correction algorithm.

Figure 23: Vertical movement, Y-axis only without algorithm corrections for RSSI and with INS algorithm corrections.

Figure 24: Vertical movement, Y-axis only with algorithm corrections for RSSI and INS

Figure 25: Sensor Fusion - Particle Filter - INS and RSSI combined, Y-axis movement, 100 particles (left), 500 particles (right).

Figure 26: Sensor Fusion - Particle Filter - INS and RSSI combined, Stationary, 500 particles..... 
Figure 27: Sensor Fusion - Particle Filter - INS and RSSI combined, Y-axis movement, 500

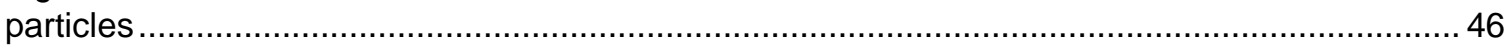

Figure 28: Sensor Fusion - Particle Filter - INS and RSSI combined, X-axis movement 


\section{CHAPTER 1 INTRODUCTION}

\subsection{Motivation}

Enhanced mechanisms and solutions to support health care is increasingly becoming one of the most challenging tasks faced by modern society. Between the combined effects of an aging baby boomer population and nursing staff shortage, the current health care system will lead to an eventual collapse [1]. This collapse is due to the fact that current hospital tasks related to patient monitoring, care, management, and supervision require nursing staff to manually execute these tasks causing an efficiency bottleneck [2]. Currently, there has been a massive nursing shortage as the average median age of nurses is 46 . More than $50 \%$ of the current nursing workforce is close to retirement. With recent reforms in healthcare, millions of people have received health care and more nurses and health professionals will be required to compensate [20]. By coming up with solutions to help automate tracking with the least amount of additional hardware such that patients in hospitals and nursing homes are the least intruded upon, better care can be provided for patients with fewer staff.

\subsection{Background and Related Works}

Wearable electronic sensing and monitoring devices have seen a rapid increase in interest over the last decade in healthcare [21]. These small wearable wireless devices have the ability to monitor and transmit biological information continuously in real-time [3]. Wireless wristbands aimed for the general public, such as the FitBit and the UP wristband by Jawbone [4] are already used to collect everyday life statistics such as sleep time and step counts. By including a method of tracking for these already available wristbands and increasing the number of sensors to include more health statistics, they can be used in nursing homes and hospitals that require more complex information such as heart rate, blood sugar, etc. as well as provide locations of patients at all times. The topic of human location tracking is of great interest in everyday applications which can generate real time information [5] at a low-cost and with the smallest intrusion level possible. Low intrusion consists of minimal user attendance; such as recharging and 
recalibration. As such, wireless sensor networks make an excellent candidate for the purpose of looking at localization.

The idea of a wireless sensor network is that of a distributed collection of nodes that are resource constrained in some form or another and capable of operating for extended periods of time without user interference.

Smartphones have become a hugely researched topic with most recent studies to monitor location with smartphones with the use of the embedded GPS radio and accelerometers [37]. Recent papers have presented detailed studies of available resources for localization on a smartphone: WiFi radio, cellular communications radio, and accelerometer [38] and have gone over room level accuracy.

\subsubsection{Inertial Motion Tracking}

The first usage of inertial navigation systems (INS) was in the 1950s in ships, submarines, and airplanes [6]. It hasn't been until recently that micromachining and electronics have pushed the boundary on low-cost small sized silicon sensors which have led to new and innovative applications in industry.

Inertial trackers are of prime importance in motion tracking applications as they are less costly, more compact, lightweight, and do not have line of sight (LOS) requirements as compared to GPS. Also, inertial trackers have low latency and can be measured at a relatively high rate (thousands of samples per second). However, the biggest problem with inertial measurement sensors is the drift problem. Drift is when errors in measurement, however small, are accumulated from point to point. This leads to an ever increasing difference between where the system thinks it is and its actual location. Error can be caused by several factors in a system these factors include [7]:

- Calibration errors in the scale factors, alignments, and linearities of the sensors. This is usually at manufacturing and cannot be easily corrected for.

Page 2 
- Constant bias $\delta \omega$ - The bias of a sensor is the average output when the device is sitting still. Constant bias errors grow linearly with time. This type of biasing is easily corrected for as you only need to take the average of the output over a long period of time while the device is not rotating. Once the constant bias is known, it can be subtracted from all measurements to eliminate the error.

- White Noise- The sensor exhibits high frequency noise that is caused by thermomechanical events. This type of noise shows up in the form of "white noise". White noise is a random amount of noise that is added to the signals that has an average amount equal to $\sigma$ and a long term average equal to zero. This can be mathematically stated as a zero mean process with standard deviation equal to $\sigma$.

- Bias Stability/Flicker Noise - The bias of the sensor will wander or walk over time due to flicker noise. The bias fluctuations due to flicker are usually modeled as a random walk.

In position estimation, when a gyroscope is included, the errors of the gyroscope are combined with the error of the accelerometer. However, since calculating position from an accelerometer requires a double integration on the acceleration, a fixed accelerometer bias error results in a position drift error that grows quadratically with time. However, though the error of the accelerometer contributes to the position estimation error, the critical errors of the system come from the orientation estimation caused by the gyroscope. In fig 1, the simulation shows variousgrade gyroscopes and their drift over a period of time. An error in the tilt orientation measurement causes an error in the horizontal component of acceleration. Because of this it is the gyroscope, not the accelerometer, which causes the most positional accuracy errors in navigation. Therefore, exploring new techniques to reduce drift in inertial systems has become the primary goal in the field of motion tracking. 


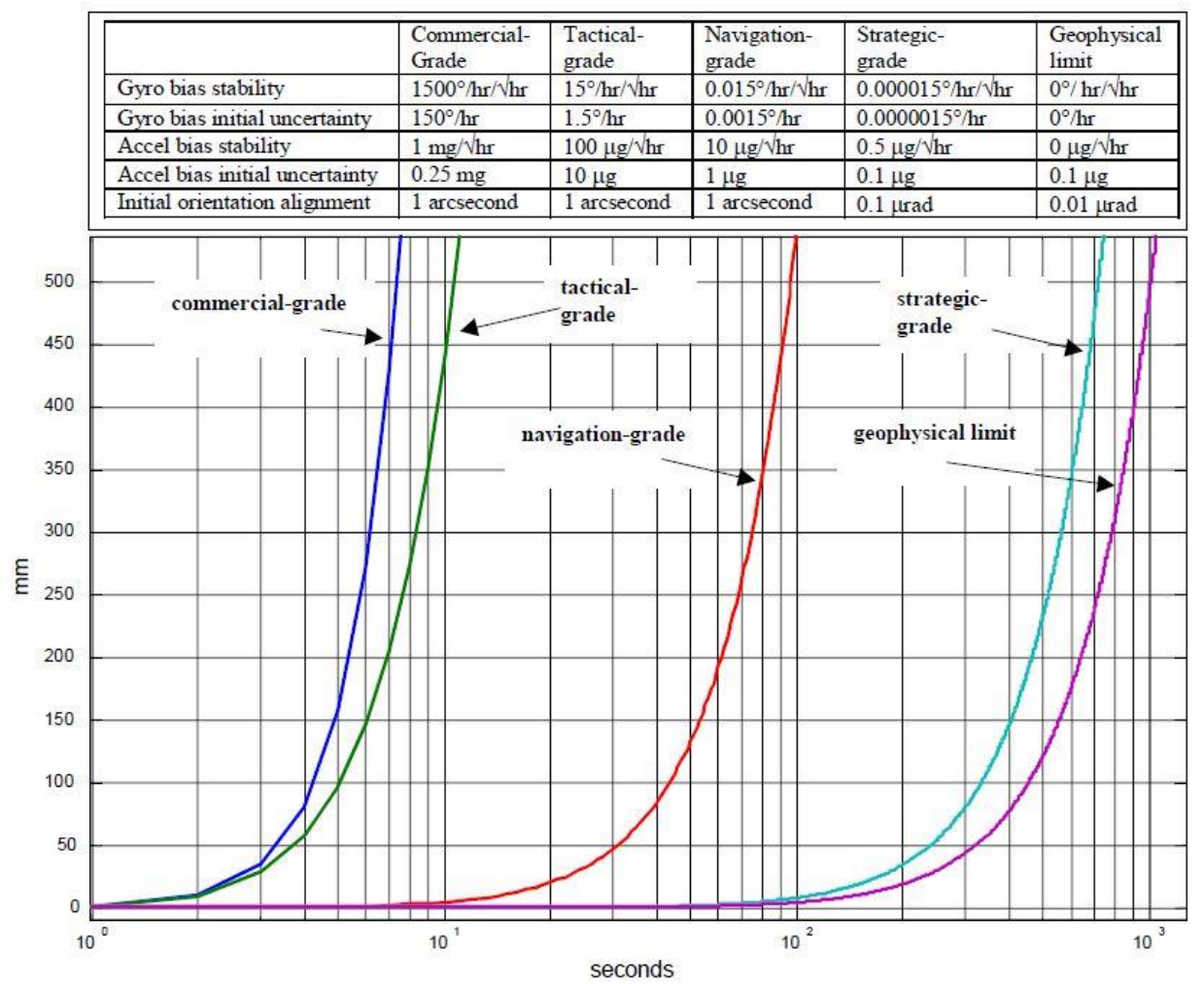

Figure 1: Comparison of 1- $\sigma$ random position drift performance of commercial, tactical, navigation, strategic-grade, and "perfect" inertial navigation systems over a 20 minute covariance simulation [8] 


\subsubsection{Reducing Error in Inertial Systems}

Error reduction problems fall into one of two categories: sensor fusion or domain specific assumptions. Error reduction is an ever researched problem, where some implementations depend on the use-case of the system itself. In this paper, use of domain specific assumptions is used to characterize the generic walking motion of a user, while also using sensor fusion to attempt to gain a more accurate representation of the user itself.

\subsubsection{Sensor Fusion}

Multiple sensors in a control system can be used to provide more information, robustness, and complementary information [9]. When two or more sensory data or data derived from sensory data are combined, the results can be better than would be possible if only the individual sensors were used. This result can be more accurate, more complete, more dependable, or refer to the result of an emerging view due to redundancy of sensed data and that one sensor may have information to correct another sensor's data. When you combine sensory data from two or more types of sensors to update the state of a system, it is called sensor fusion. In an inertial system, the state usually consists of position, velocity, and orientation of the object. A sensor fusion algorithm calculates the state using inertial sensors signals together with signals from addition sources such as magnetometers [10].

Using magnetometers and gyroscopes together is an example of how using sensor fusion can give better results than using sensors independently. Magnetometers can be used to correct for gyroscope drift using sensor fusion. Magnetometers measure the magnetic field's strength and direction allowing the north direction to be found. While the magnetometers are able to calculate orientation, the magnetometer is susceptible to interference from ferromagnetic materials which distort the orientation measurement. Thus, magnetometers are not able to be a replacement for the gyroscope. However, when combining both the gyroscope and the magnetometer together, the accuracy of the calculation for the orientation can be improved through redundancy and correct for drift. 


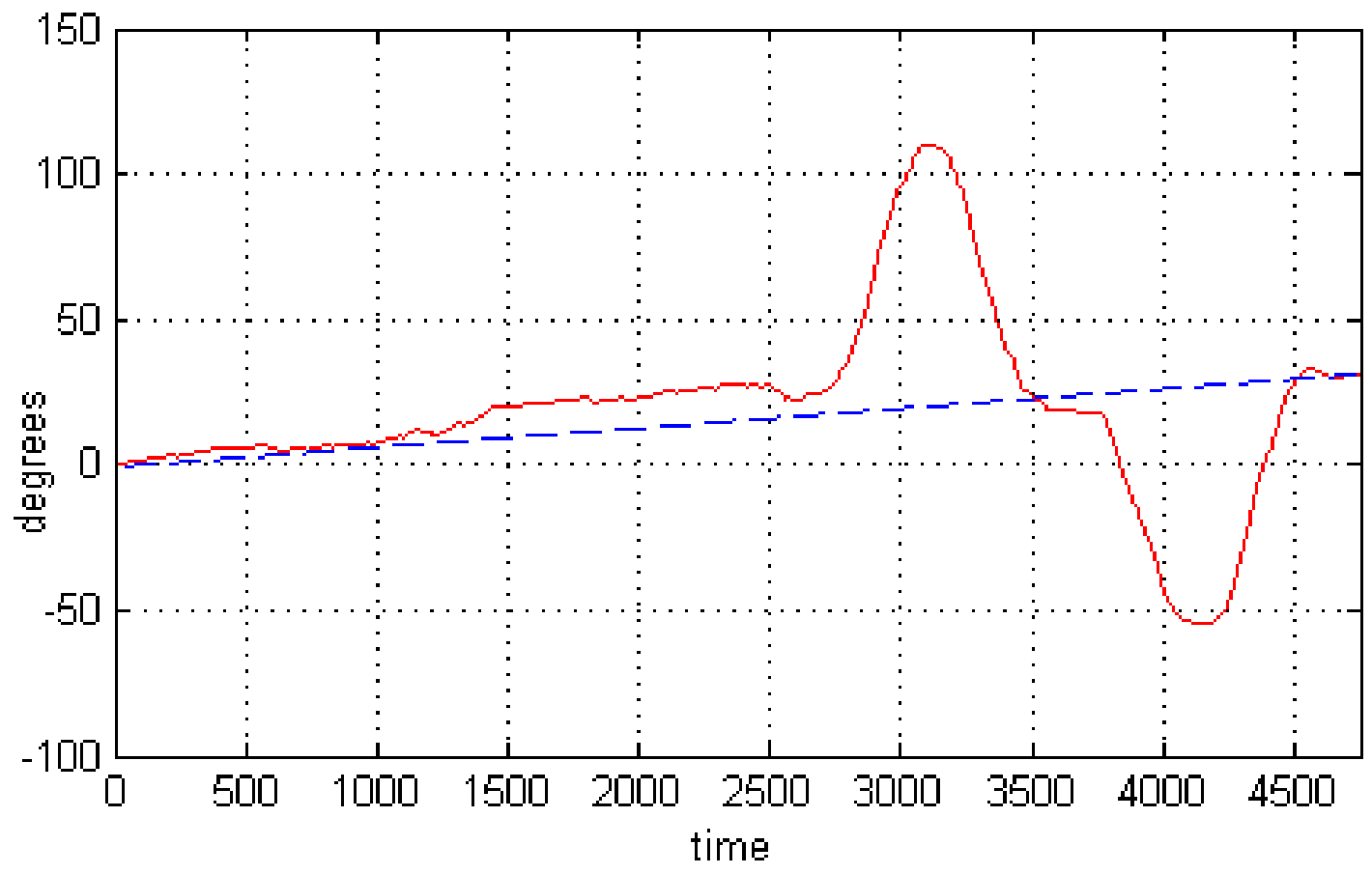

Figure 2: Example of gyroscope drift over time. Blue line indicates drift over time, red indicates the value of the gyroscope. [29]

Figure 2 shows an example of gyroscope drift over time that occurs within a system. While magnetometers will help correct for gyroscope drift, it is also possible to use an accelerometer to correct for the drift as well for the pitch and roll.

It is important to show the drift problem in its entirety as the calculation for $x, y$ positional change can be represented as the sine and cosine of the current gyroscope calculation multiplied by the positional change given by the accelerometer. A drift can cause your positional change to affect unintended axes.

Several mathematical models are currently used to fuse sensor data together for tracking. These methods include the Kalman filter, the various offshoots of the Kalman filter, such as the Extended Kalman, Unscented Kalman, and Complementary Kalman, and the Particle Filter with current tracking techniques generally using an Extended Kalman Filter (EKF) for sensor 
fusion. Note, that in current tracking problems, an application of sensor fusion includes GPS/INS where information from both the Global Position System and Inertial Navigation System are used to fuse data together in a mathematical model like the Extended Kalman Filter. [39]

For the purpose of this thesis, sensor fusion occurred with position estimation from RSSI and the INS information. Much like GPS/INS, it takes many aspects and uses them to attempt to do RSSI/INS while keeping constraints.

By combining these mathematical models together with multiple forms of data, a more accurate representation of position estimation can occur.

\subsubsection{Domain Specific Assumptions}

Domain specific assumptions are used in applications where specific assumptions can be made about movement to reduce positional error. For instance, during movement, a human has a stationary stance and a movement stance [12]. The stationary stance can easily be distinguished from the movement stance. This allows for the correction of drift and positional error that has accumulated during the period of time during movement. By measuring the acceleration due to gravity during the stationary phase, inclination errors can also be corrected for. For instance, the paper of Bachman [11] a Kalman filter is used for inertial tracking. The filter corrects for the drift based on the domain specific assumption that the limb acceleration is bounded and will average to zero for an extended period of time. The paper of Zhou and Hu [13] developed a kinematic model of upper limb movement to remove undesired biases and noise. By using this model they combine inertial sensors together using a Complementary Kalman filter to track human movement.

The work in this research uses both of the previously discussed correction techniques in inertial systems. It uses domain specific assumptions for the positional estimation. It assumes that the acceleration of the user will consistently be front-facing, with rare situations causing a movement in the $-x$ direction. This estimation is then used by the filter along with the RSSI values to continuously reduce the positional error of both the INS and the RSSI information. Sensor 
fusion is used to combine the inertial systems with the RSSI measurements via the filter as well to give a more accurate measurement of position. Through this, a more complete solution is formed and usable for motion tracking. For this paper, instead of using a Kalman Filter for the specific applications, a particle filter (Sequental Monte Carlo) is used instead.

\subsubsection{RSSI-Based Tracking}

Received Signal Strength Indicator (RSSI) is used to get an estimate of the position between a transmitter and receiver (ranging). Transmitters which know their coordinates and have known locations can be used to calculate a moving nodes position. These transmitters are known as reference nodes, or anchor nodes. RSSI based techniques are generally used for indoor localization which is used to locate an object or person within a building. There is a major disadvantage with RSSI due to the multipath interference that occurs from the obstruction of furniture, walls, and other building specific obstacles. This multipath interference at the receiving antenna causes range estimation errors that translate to large positioning errors [14]. Despite this disadvantage, RSSI-based tracking is intensively studied for the purposes of indoor tracking and positioning due to the limitation of other positioning techniques for indoor localization such as Time Delay of Arrival (TDOA) and Time of Arrival (TOA) systems. For a less intrusive system, TDOA and TOA cannot be used as they require an extra physical layer to use. Compared to these other systems, RSSI-based tracking is low cost, low energy, and can be deployed with any wireless sensor network since RSSI is natively supported by most existing transistor chipsets with no extra hardware costs [15]. This makes it an excellent candidate for future works involving smart phone localization and other such topics.

RSSI-based localization algorithms can be classified into one of two categories: range-based and range-free algorithms. Range-based algorithms use the RSSI signal to calculate the distance from the anchor node of known position. It then uses methods such as multilateration or triangulation to calculate the position of the unknown node's position. These methods require two or more anchor nodes at fixed and known locations to calculate position. With the more nodes, the more accurate the position estimation. The method of range-based algorithms is the most 
susceptible to multipath interference and shadowing effects and thus has limited accuracy regardless of the number of nodes. Range-free algorithms do not calculate the RSSI value to compute distance but instead uses it in other ways. For instance, the RSSI value is interpreted from a pre-calculated RSSI map. A preliminary accurate mapping of RSSI values in each position of the map is required. Comparing the RSSI values received from different anchors with a prebuilt RSSI map, a node can estimate its own position in the area [10].

RSSI-based tracking systems generally have an accuracy within a meter[16]. This has caused RSSI-based tracking to not be a viable solution for the motion tracking problem as it requires much higher accuracy. However, Blumrozen et al. [17] suggested and showed that under certain conditions, RSSI can be a valid solution for the human motion tracking problem. They achieved accuracy of several centimeters.

Since RSSI is readily deployable with no extra hardware requirement. It is an excellent candidate for sensor fusion with an inertial system. This research uses a particle filter to combine the described RSSI system with the inertial system's data to solve the human tracking problem indoors.

\subsection{Thesis Outline}

The thesis is organized as follows:

Chapter 2: Introduces the system model of the gyroscope, accelerometer, and RSSI

Chapter 3: Discusses the concept behind trilateration estimation using RSSI and the particle filter. Chapter 4: Describes the experimental setup and methods used to collect data.

Chapter 5: Provides results and analysis of the solution.

Chapter 6: Provides discussion and future works. 


\section{CHAPTER 2 SYSTEM MODEL}

\subsection{System Description}

Figure 3 shows the system setup and its components. The system consists of $\mathrm{N}$ anchor nodes and one mobile node. Each node is equipped with a wireless transceiver such that it can transmit data between them and communicate with the base station. The anchor nodes transmit data to the mobile node so the mobile node can calculate the RSSI value. This value is then transmitted to the base station with all the sensor data of the mobile node so that the calculations can be done for the position of the mobile node. The sensors equipped to the mobile node include a gyroscope and accelerometer which help record the node's inertial data for an inertial navigation system. The goal of this work is to continuously monitor the node's location using the inertial data of the sensors and the RSSI attenuation measured by using the anchor nodes.

The research is aimed at providing an analysis of method of location estimation indoors for tracking and monitoring. Much of this chapter is thanks to the thesis this work is based off of [10]. 


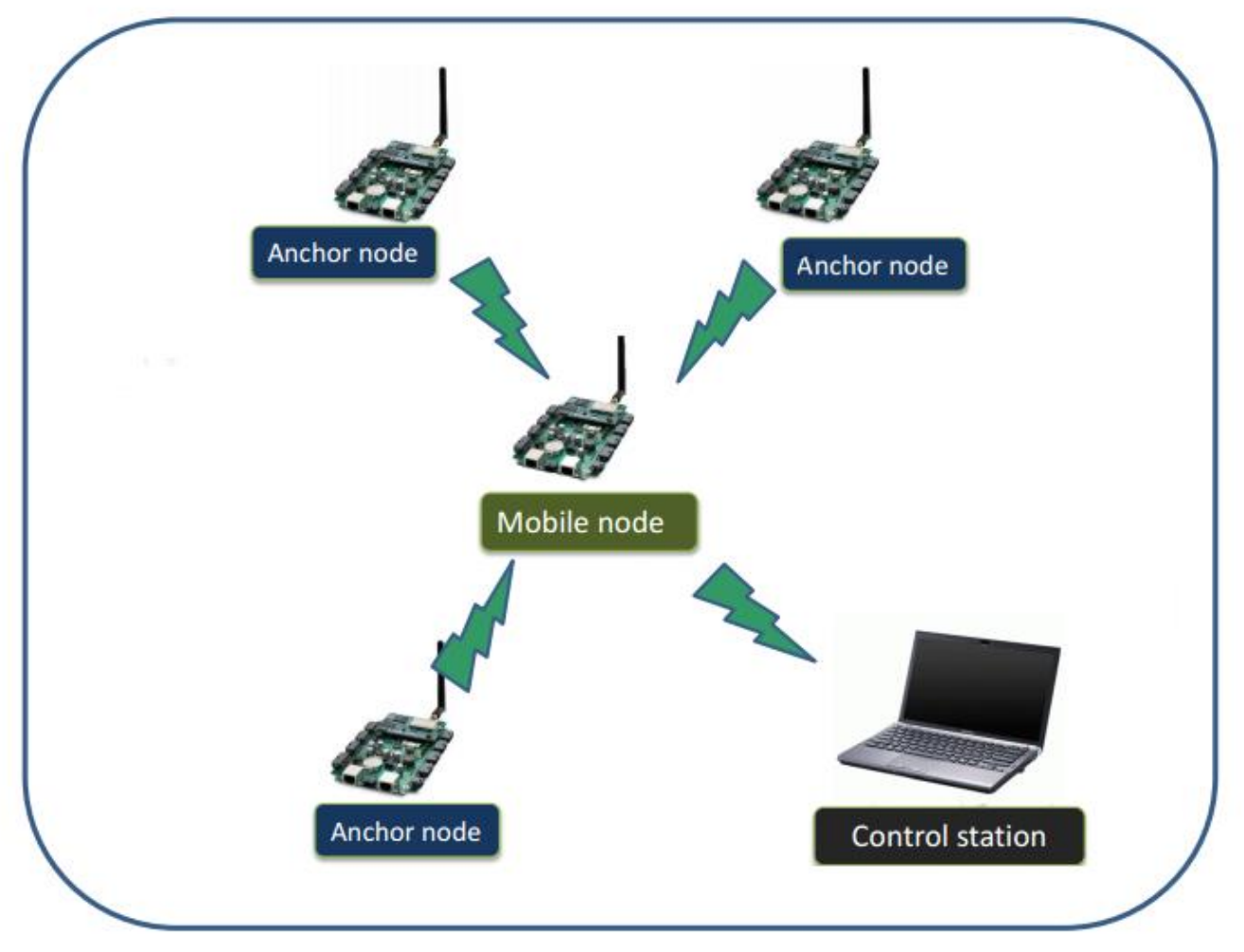

Figure 3: [10] System description diagram. The diagram shows the mobile node, the anchor nodes, and the control station (or base station) and the relationship between them. The anchor nodes send packets to the mobile node, and the mobile node sends all resulting information from the gyroscope, accelerometer, and RSSI information to the control station. The control station aggregates, and estimates location utilizing both the inertial measurements and RSSI-based estimates.

\subsection{Inertial Sensor Modeling}

A three axis accelerometer and gyroscope sensor compose the system. For drift correction on the gyroscope, a magnetometer can be added to the system in future works to further improve results [18]. Figure 4 shows the sensor model diagram. The diagram describes each signal and the relationships between them. 


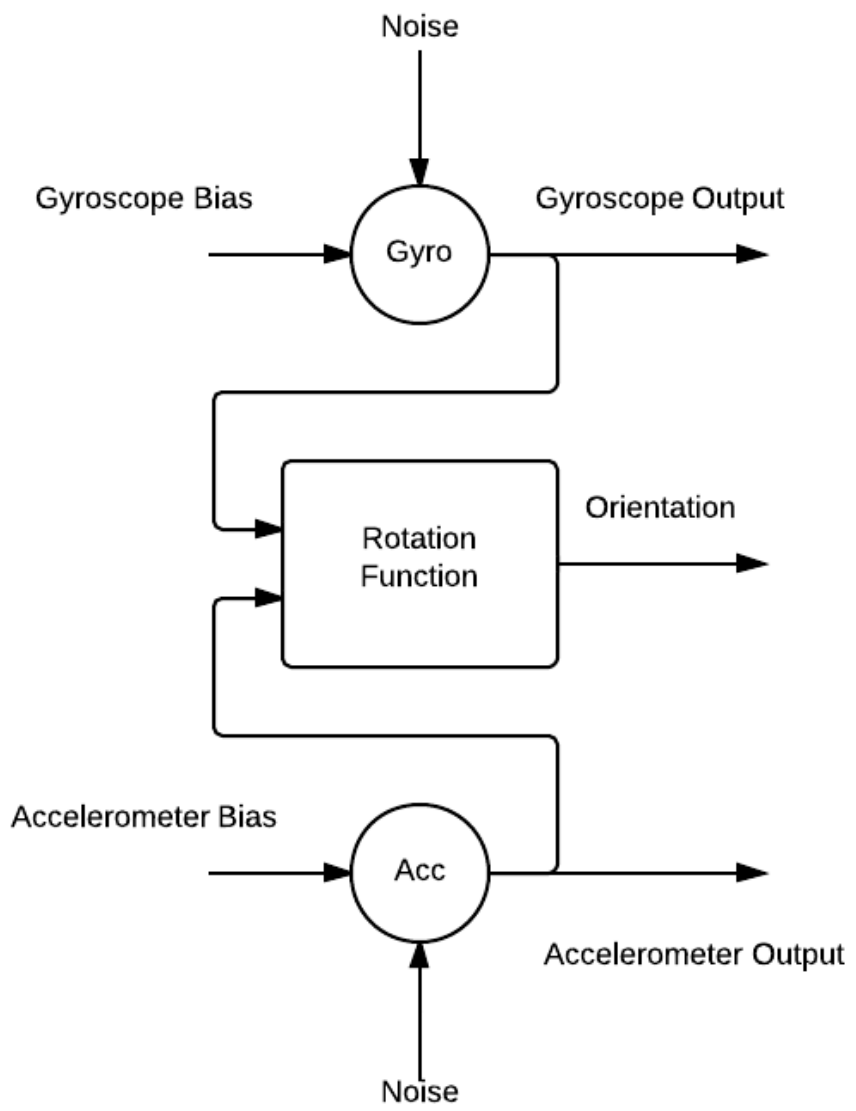

Figure 4: The sensor model diagram and its relations. The diagram details the inputs and outputs of the sensors used on the mobile node and their interaction between each other. Gaussian white noise is added to each of the signals, and a slight biasing signal is applied to the gyroscope upon startup. The orientation is a combination of the accelerometer and gyroscope to correct for gyroscope drift.

The gyroscope and accelerometer are used in conjunction with eachother to calculate the

\subsubsection{Gyroscope Model}

The gyroscope model can be defined as the gyroscope bias $b_{g, t}$, the angular velocity $\omega_{t}$, and $a$ combination of white noise measurement $v_{G, t}[22]$. For the following equations, the subscript $g$ denotes the gyroscope.

$$
\mathrm{y}_{\mathrm{G}, \mathrm{t}}=\omega_{\mathrm{t}}+\mathrm{b}_{\mathrm{g}, \mathrm{t}}+\mathrm{v}_{\mathrm{G}, \mathrm{t}}
$$

The white noise process can be modeled as 


$$
\mathrm{v}_{\mathrm{G}, \mathrm{t}} \sim \mathrm{N}\left(0, \sigma_{\mathrm{g}, \mathrm{t}}^{2}\right)
$$

It is important to note that the slow variation offset bias that the gyroscope experiences is modeled as a realization of a first order Markov process which is driven by a white Gaussian noise vector $\mathrm{w}_{\mathrm{g}, \mathrm{t}}$.

$$
b_{g, t}=b_{g, t-1}+w_{g, t}
$$

\subsubsection{Accelerometer Model}

The accelerometer model can be described as the sum of the acceleration $a_{t}$, the gravity vector, $g_{t}$, the small sensor bias $b_{a, t}$, and the white Gaussian noise $v_{A, t}$

$$
\mathrm{y}_{\mathrm{A}, \mathrm{t}}=\mathrm{a}_{\mathrm{t}}-\mathrm{g}_{\mathrm{t}}+\mathrm{b}_{\mathrm{a}, \mathrm{t}}+\mathrm{v}_{\mathrm{A}, \mathrm{t}}
$$

Where the white noise process for the acceleration can be modeled as

$$
\mathrm{v}_{\mathrm{A}, \mathrm{t}} \sim \mathrm{N}\left(0, \sigma_{\mathrm{a}, \mathrm{t}}^{2}\right)
$$

The accelerometer bias is similar to the gyroscope as it is a realization of a first order Markov process driven by a white Gaussian noise vector $\mathrm{w}_{\mathrm{a}, \mathrm{t}}$.

$$
\mathrm{b}_{\mathrm{a}, \mathrm{t}}=\mathrm{b}_{\mathrm{a}, \mathrm{t}-1}+\mathrm{w}_{\mathrm{a}, \mathrm{t}}
$$




\section{CHAPTER 3 BACKGROUND CONCEPTS}

\subsection{RSSI Trilateration}

Trilateration is used to find an unknown location from at least three known locations. Trilateration uses the distances from the known locations to estimate the coordinate of the unknown location. The distances between reference locations and the unknown location can be treated as the radii of many circles with centers at every reference location. Thus, unknown location is the intersection of all the spherical surfaces as shown in figure 5. [24]

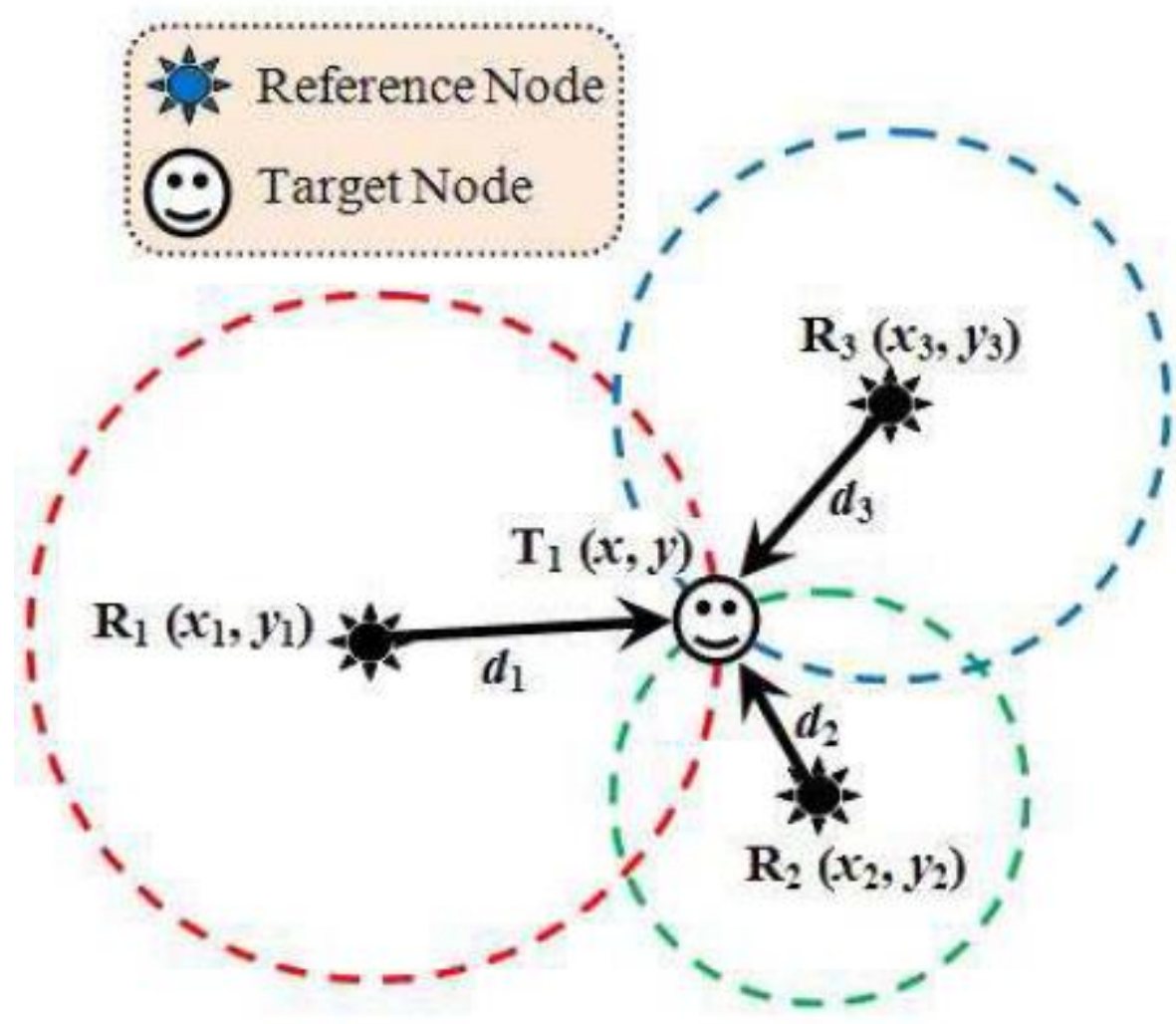

Figure 5: Trilateration Estimation [26] which shows the concentric circles used by the RSSI ranging technique to calculate the position using $R_{1}, R_{2}, R_{3}$. The mobile node, $T_{1}$, is at the centerpoint of all three circles of each anchor node.

In Fig. 5, there are three nodes that are randomly allocated with known location $R 1\left(x_{1}, y_{1}\right), R 2\left(x_{2}\right.$, $\left.y_{2}\right), R 3\left(x_{3}, y_{3}\right)$. Node $T_{1}(x, y)$ at unknown coordinates can be calculated using reference nodes $R 1$, $R 2$, and R3 and the distances $d_{1}, d_{2}$, and $d_{3}$ between the reference nodes and the unknown target node, $T_{1}$. A solution to this problem can be achieved using the Pythagorean Theorem [26]. 


$$
\begin{aligned}
& d_{1}^{2}=\left(x_{1}-x\right)^{2}+\left(y_{1}-y\right)^{2} \\
& d_{2}^{2}=\left(x_{2}-x\right)^{2}+\left(y_{2}-y\right)^{2} \\
& d_{3}^{2}=\left(x_{3}-x\right)^{2}+\left(y_{3}-y\right)^{2}
\end{aligned}
$$

Rearranging the equations in (3.1) and solving for $x$ and $y$. [26]

$$
\begin{aligned}
& \mathrm{x}=\frac{\mathrm{AY}_{32}+\mathrm{BY}_{13}+\mathrm{CY}_{21}}{2\left(\mathrm{x}_{1} \mathrm{Y}_{32}+\mathrm{x}_{2} \mathrm{Y}_{13}+\mathrm{x}_{3} \mathrm{Y}_{21}\right.} \\
& \mathrm{y}=\frac{\mathrm{AX}_{32}+\mathrm{BX}_{13}+\mathrm{CX}_{21}}{2\left(\mathrm{y}_{1} \mathrm{X}_{32}+\mathrm{y}_{2} \mathrm{X}_{13}+\mathrm{y}_{3} \mathrm{X}_{21}\right.}
\end{aligned}
$$

Where [26]

$$
\begin{aligned}
& A=x_{1}{ }^{2}+y_{1}{ }^{2}-d_{1}{ }^{2} \\
& B=x_{2}{ }^{2}+y_{2}{ }^{2}-d_{2}{ }^{2} \\
& C=x_{3}{ }^{2}+y_{3}{ }^{2}-d_{3}{ }^{2}
\end{aligned}
$$

and

$$
\begin{aligned}
& X_{32}=\left(x_{3}-x_{2}\right) \\
& X_{13}=\left(x_{1}-x_{3}\right) \\
& X_{21}=\left(x_{2}-x_{1}\right) \\
& Y_{32}=\left(y_{3}-y_{2}\right) \\
& Y_{13}=\left(y_{1}-y_{3}\right) \\
& Y_{21}=\left(y_{2}-y_{1}\right)
\end{aligned}
$$

By using equations 3.2 and 3.3 in conjunction with 3.4 and 3.5 , localization is easily achieved because the distances $\left(d_{1}, d_{2}, d_{3}\right)$ can be obtained from ranging. The locations of each of the reference nodes $\left(x_{1}, y_{1}\right),\left(x_{2}, y_{2}\right)$, and $\left(x_{3}, y_{3}\right)$ can be stored at the reference node itself, or at a base station. In large scale networks, three possible scenarios for localization might occur: 
1. The node(s) is able to reach at least three reference nodes.

2. The node(s) is able to reach one or two reference nodes only.

3. The node(s) is unable to reach any reference node at all.

For any localization system, three reference nodes are required. Therefore, it is important to set up the system such that at any given point, three reference nodes can be reached.

\subsubsection{RSS Ranging in Indoor Environment}

All electromagnetic waves have an inverse-square relationship between power received and distance. Therefore, the strength of the power from a received signal can be used to estimate the distance. [27] The following expression is an example of this:

$$
\mathrm{P}_{\mathrm{r}} \propto \frac{1}{\mathrm{~d}^{2}}
$$

Where $\mathrm{P}_{\mathrm{r}}$ is the received power at distance $d$ from the transmitter. This expression states that the distance of the signal traveled can be found by comparing the received power and transmitted power, also known as path loss. [24]

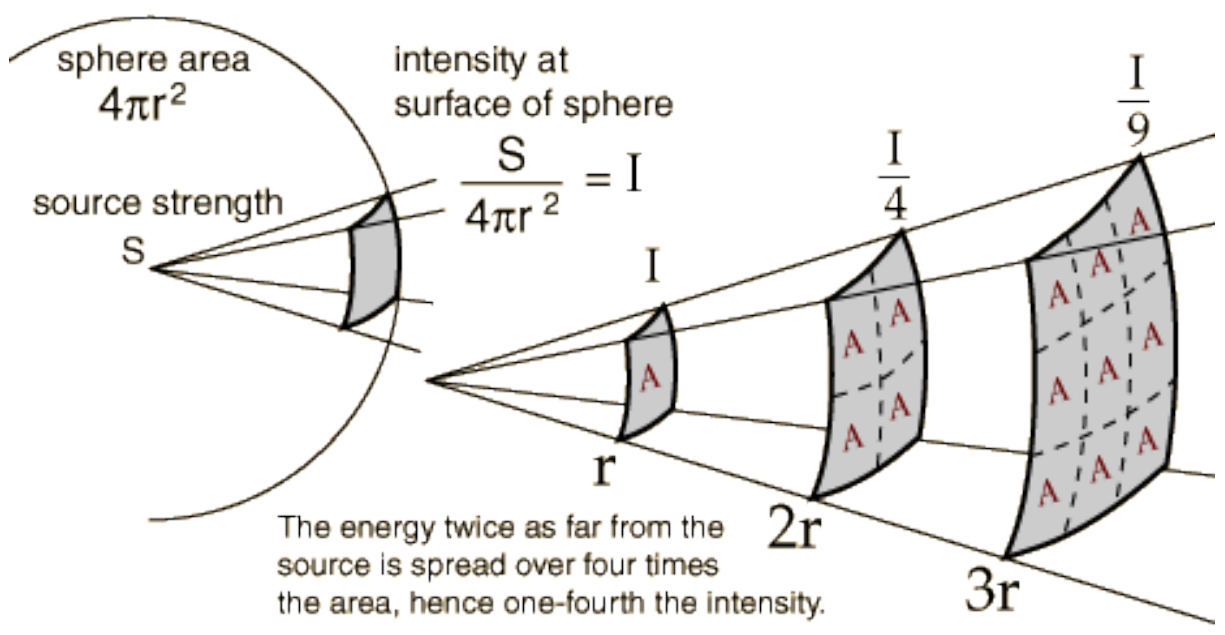

Figure 6: Inverse Square Law, general. [30] 
In a realistic environment, the increment of the path loss due to the distance may be different in different environments. For this reason, it is useful to characterize the environment using path loss exponent $\mathrm{n}$ as shown in the expression [24]:

$$
\mathrm{P}_{\mathrm{r}}=\frac{\mathrm{P}_{(\mathrm{d} 0)}}{\left(\frac{\mathrm{d}}{\mathrm{d} 0}\right)^{\mathrm{n}}}
$$

Where $P_{\text {Ido) }}$ is the received power at distance $d_{0}$. The value of $d_{0}$ is fixed at a constant, usually, of $1 \mathrm{~m}$. The value of the path loss exponent $\mathrm{n}$ increases if the increment of the path loss is more drastic when distance increases. This is seen in figure 7 below.

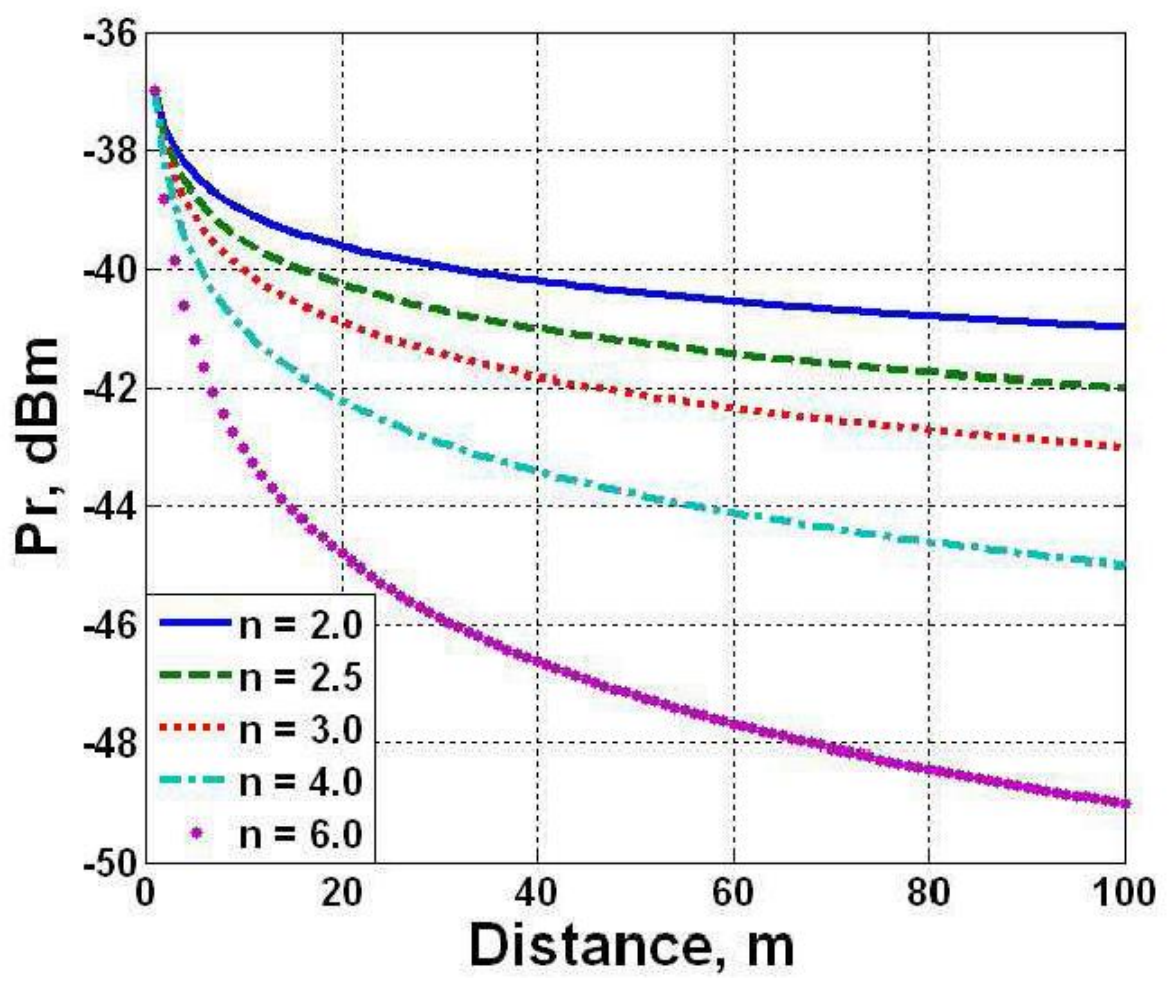

Figure 7: Effects of path exponent [24]

Figure 7 shows an important feature that is a rule of path loss. At $0 \mathrm{~m}$, no matter what path loss value $\mathrm{n}$ is, it starts at the same $\mathrm{dBm}$ value. In the instance of this graph, the starting point of each curve is at $-37 \mathrm{dBm}$. Even if the setting was smaller or higher, all curves would be be up or down 
shifted. This shows the importance of $\mathrm{P}_{(\mathrm{d} 0)}$ as an important parameter to characterize environment. [24].

\subsubsection{RSSI System Design}

Location system design requires a setup characterization of each transmitting stationary beacon such that the system can provide meaningful data. In order to do this several processing steps, as shown in Fig. 8, must be done.

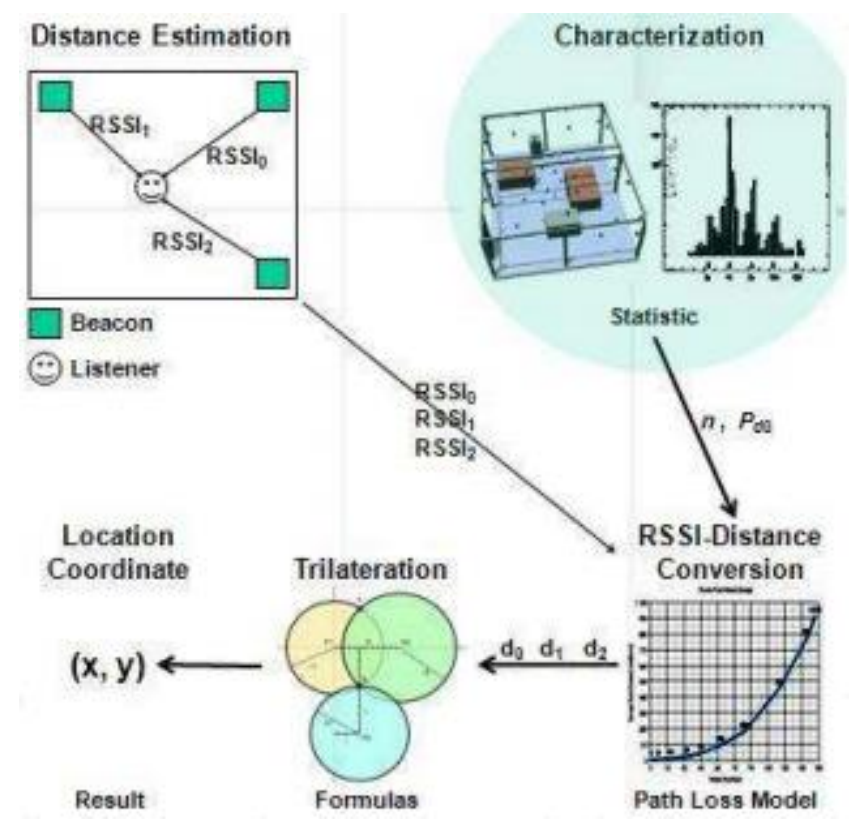

Figure 8: The Findings of Location from Raw RSSI [26]

RSSI values are collected during the distance estimation step from stationary beacon nodes transmitting to the listener node. These nodes are placed at known locations and then used to characterize the environment for suitable parameters in the area being operated in.

Once the environmental characterization has been completed, continuous RSSI measurements can be taken in from the stationary nodes to the listener such that the values can be converted into a distance estimation using the path loss model. Once these node distances have been successfully found, we have the distances of each node to the listener. By applying trilateration to 
the nodes, it combines the distance measurements to find the exact location coordinates of the sensor node. The overall block diagram for these steps can be found in Fig. 9.

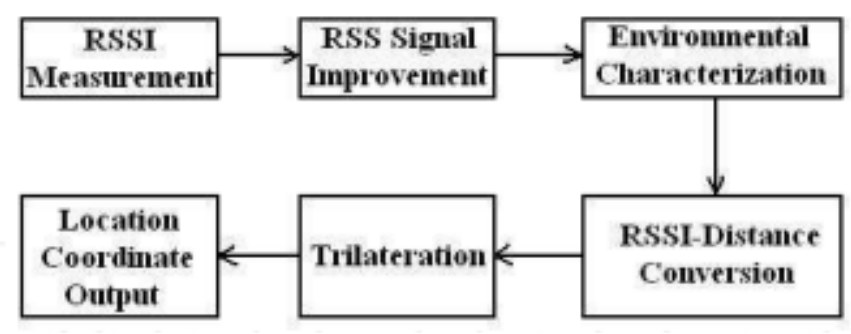

Figure 9: Overall System Block Diagram [26]

\subsubsection{RSSI Environmental Characterization}

It's possible to calibrate the system parameters to the most ideal values. After the environmental characterization step, the distance estimation of the signal is adjusted to the minimum state error [24].

As shown in figure 7 , the value of the path loss exponent $n$ affects the power received at certain distances. This provides a good basis for our environmental characterization step.

"The important parameters used to characterize environment include path loss exponent $\mathrm{n}$ and the received power $\operatorname{Pr}(\mathrm{d} 0)$ measured at distance $\mathrm{d} 0$ to the transmitter. For each enclosed area of indoor environment, a pair of these parameters $(n, \operatorname{Pr}(\mathrm{d} 0))$ are used to represent the conditions of the area. To characterize the area for RSS ranging, received power $\operatorname{Pr}(\mathrm{d} 0)$ is first measured by allocating a receiver $d 0$ apart from the transmitter. $d_{0}$ is generally fixed at 1 meter. After $\operatorname{Pr}(\mathrm{d} 0)$ is obtained, the receiver is moved to other locations to measure path loss exponent $\mathrm{n}$ using the following expression:" [24]

$$
n=\frac{P_{r(d 0)}-P_{r(d)}}{10 \times \log _{10}\left(d / d_{0}\right)}
$$

Where $P_{r(d)}$ is the received power of the receiver measured at a distance $d$ to the transmitter, which is expressed in $\mathrm{dBm}$. Theoretically, every room has their own environmental parameters. Therefore, every room must be characterized if used in the system. Also, it is possible for two 
neighbors within the same room to have different characterizations within the system. This increases the difficulty of environmental calibration works. Environmental characterizations can also change if objects within the room shift or are added.

\subsubsection{RSSI-Distance Conversion}

Once the environmental characterization step is done, it is necessary to convert RSSI to distance.

If RSS ranging is used to measure the distance between reference nodes and the target node, then the log-distance path loss model [28] is used to express the relationship between the received power and the distance as shown in the following equation [24]:

$$
P_{r(d)}=P_{r(d 0)}-10 \times \log _{10}\left(\frac{d}{d_{0}}\right)
$$

After the setup of environmental characterization, the value of $n$ and $P_{r(d 0)}$ have been calculated and obtained. Thus, the distance between transmitter and receiver can be estimated using the following expression with the current power received at unknown distance $\mathrm{P}_{\mathrm{r}(\mathrm{d})}$ :[24]

$$
d=d_{0} 10^{\left(\frac{P_{r(d 0)^{-P}} r(d)}{10 n}\right)}
$$

Where the value of $d$ is in the same units as the value of $d_{0}$. 


\subsection{Particle Filter}

A Bayesian filter is used to estimate the state of any system that changes over time using a sequence of noisy measurements made on the system. The particle filter is a way to implementing the recursive Bayesian filter by Monte Carlo simulations. The key feature here is to represent the required posterior density function by a set of random samples with associated weights and to compute estimates based on these samples and weights. [34]. Section 3.2.2 goes into the nomenclature that will be needed to understand this section, and 3.2.3 will go over the mathematics of the Sequential Importance Sampling (SIS) algorithm used in this thesis.

\subsubsection{Particle Filter Nomenclature}

In probability theory, a random measure is a measure-valued random element. While a random variable is a variable whose value is real and subject to mathematical randomness, or chance, a random element is a more generalized representation of a random variable such that instead of associating the value with a real number, it can be associated with functions, vectors, processes, sets, collection of sets, transformations, etc.

In Bayesian estimation or statistics, the posterior probability is the conditional probability of a random event of an uncertain proposition that has been assigned after relevant observations have occurred and been used to calculate its probability. Related is the posterior probability distribution which is treated as a random variable conditional on (given two jointly distributed $\mathrm{X}$ and $Y$ random variables, the probability of $X$ given when $Y$ is at some particular value) the observation obtained from the experiment or survey itself.

With statistics, the idea of probability density functions or densities is a function that describes the chance or likelihood of a random variable taking on a given value.

\subsubsection{Particle Filter Sequential Importance Sampling}

The sequential importance sampling particle filter is used to represent the posterior density function as a random set of samples with weights and then compute estimates based on these samples and weights. With an increased number of samples, the Monte Carlo simulation becomes a close approximation to the usual functional description of the posterior pdf and, as 
such, the particle filter approaches the optimal estimate of the state. Much of this section's math is thanks to the work of [34].

"Let us specify that the posterior density can be approximated as:

$$
p\left(x_{0: k} \mid z_{1: k}\right) \approx \sum_{i=1}^{N_{s}} w_{k}^{i} \delta\left(x_{0: k}-x_{0: k}^{i}\right)
$$

Since the posterior density can be approximated by $p\left(x_{0: k} \mid z_{1: k}\right)$, we have a discrete weighted approximation to the true posterior. The weights are chosen using the principle of importance sampling [35], [36]. The principle relies on the following. Suppose $p(x) \propto \pi(x)$ is a probability density for which it is hard to draw samples from, but it is possible for $\pi(x)$ to be evaluated. This also allows for $\mathrm{p}(\mathrm{x})$ to a certain extent as well due to proportionality. Also, let $\mathrm{x}^{\mathrm{i}} x^{i} \sim q(x), i=$ $1, \ldots, N_{s}$ be samples that are generated from a proposal $q($.$) which is called an importance$ density. A weighted approximation to the density $p($.$) is given by:$

$$
p(x) \approx \sum_{i=1}^{N_{S}} w^{i} \delta\left(x_{0: k}-x_{0: k}^{i}\right)
$$

Where

$$
w^{i} \propto \frac{\pi\left(x^{i}\right)}{q\left(x^{i}\right)}
$$

Is the normalized weight of the ith particle and $\mathrm{N}_{\mathrm{s}}$ is the number of samples.

Therefore, if the samples $x_{0: k}^{i}$ where drawn from an importance density $q\left(x_{0: k} \mid z_{1: k}\right)$, then the weights in (3.11) are defined by (3.13) to be:

$$
w_{k}^{i} \propto \frac{p\left(x_{0: k}^{i} \mid z_{1: k}\right)}{q\left(x_{0: k}^{i} \mid z_{1: k}\right)}
$$

Returning to the sequential case, at each iteration, one could have samples constituting an approximation to $p\left(x_{0: k-1} \mid z_{1: k-1}\right)$ and want to approximate $p\left(x_{0: k} \mid z_{1: k}\right)$ with a new set of samples. If the importance density is chosen to factorize such that 


$$
q\left(x_{0: k \mid} z_{1: k}\right)=q\left(x_{k} \mid x_{0: k-1}, z_{1: k}\right) q\left(x_{0: k-1} \mid z_{1: k-1}\right)
$$

Then one can obtain samples $x_{0: k}^{i} \sim q\left(x_{0: k} \mid z_{1: k}\right)$ by augmenting each of the existing samples $x_{0: k-1}^{i} \sim q\left(x_{0: k-1} \mid z_{1: k-1}\right)$ with the new state $x_{k}^{i} \sim q\left(x_{k} \mid x_{0: k-1}, z_{1: k}\right)$. To derive the weight update equation, $p\left(x_{0: k} \mid z_{1: k}\right)$ is first expressed in terms of $p\left(x_{0: k-1} \mid z_{1: k-1}\right), p\left(z_{k} \mid x_{k}\right)$, and $p\left(x_{k} \mid x_{k-1}\right)$.

$$
\begin{gathered}
p\left(x_{0: k} \mid z_{1: k}\right)=\frac{p\left(z_{k}\left|x_{0: k}\right| z_{1: k-1}\right) p\left(x_{0: k} \mid z_{1: k-1}\right)}{p\left(z_{k} \mid z_{1: k-1}\right)} \\
=\frac{p\left(z_{k}\left|x_{0: k}\right| z_{1: k-1}\right) p\left(x_{k}\left|x_{0: k-1}\right| z_{1: k-1}\right)}{p\left(z_{k} \mid z_{1: k-1}\right)} \\
\quad \times p\left(x_{0: k-1} \mid z_{1: k-1}\right) \\
=\frac{p\left(z_{k} \mid x_{k}\right) p\left(x_{k} \mid x_{k-1}\right)}{p\left(z_{k} \mid z_{1: k-1}\right)} p\left(x_{0: k-1} \mid z_{1: k-1}\right) \\
\propto p\left(z_{k} \mid x_{k}\right) p\left(x_{k} \mid x_{k-1}\right) p\left(x_{0: k-1} \mid z_{1: k-1}\right)
\end{gathered}
$$

By substituting (3.15) and (3.17) into (3.14), the weight update equation can be shown to be

$$
\begin{aligned}
w_{k}^{i} & \propto \frac{p\left(z_{k} \mid x_{k}^{i}\right) p\left(x_{k}^{i} \mid x_{k-1}^{i}\right) p\left(x_{0: k-1}^{i} \mid z_{1: k-1}^{i}\right)}{q\left(x_{k}^{i} \mid x_{0: k-1}^{i}, z_{1: k}\right) q\left(x_{0: k-1}^{i} \mid z_{1: k-1}\right)} \\
& =w_{k-1}^{i} \frac{p\left(z_{k} \mid x_{k}^{i}\right) p\left(x_{k}^{i} \mid x_{k-1}^{i}\right)}{q\left(x_{k}^{i} \mid x_{0: k-1}^{i}, z_{1: k}\right)}
\end{aligned}
$$

Furthermore, if $q\left(x_{k} \mid x_{0: k-1}, z_{1: k}\right)=q\left(x_{k} \mid x_{k-1}, z_{k}\right)$, then the importance density becomes only dependent on $x_{k-1}$, and $z_{k}$. This is useful in the common case when only a filtered estimate of $p\left(x_{k} \mid z_{1: k}\right)$ is required at each time step. In such scenarios only $x_{k}^{i}$ need to be stored; therefore, one can discard the path $x_{0: k-1}^{i}$ and history of observations $z_{1: k-1}$. The modified weight is then 


$$
w_{k} \propto w_{k-1}^{i} \frac{p\left(z_{k} \mid x_{k}^{i}\right) p\left(x_{k}^{i} \mid x_{k-1}^{i}\right)}{q\left(x_{k}^{i} \mid x_{0: k-1}^{i}, z_{1: k}\right)}
$$

and the posterior filtered density $\mathrm{p}\left(\mathrm{x}_{\mathrm{k}} \mid \mathrm{z}_{1: \mathrm{k}}\right)$ can be approximated as

$$
\mathrm{p}\left(\mathrm{x}_{\mathrm{k}} \mid \mathrm{z}_{1: \mathrm{k}}\right) \approx \sum_{\mathrm{i}=1}^{\mathrm{N}_{\mathrm{s}}} \mathrm{w}_{\mathrm{k}}^{\mathrm{i}} \delta\left(\mathrm{x}_{\mathrm{k}}-\mathrm{x}_{\mathrm{k}}^{\mathrm{i}}\right)
$$

where the weights are defined in (3.19). It can be shown that $N_{s} \rightarrow \infty$, the approximation (3.20) approaches the true posterior density $\mathrm{p}\left(\mathrm{x}_{\mathrm{k}} \mid \mathrm{z}_{1: \mathrm{k}}\right)$.

The algorithm used thus consists of recursive propagation of the weights and support points as each measurement is received sequentially." [34]

The particle filter for the experiment is described in chapter 4 . 


\section{CHAPTER 4 EXPERIMENTAL SETUP}

Experimental configurations were determined to properly analyze the system and the defined problem. The experiments were carried out in a $3 \mathrm{~m} \times 4 \mathrm{~m}$ room in an indoor environment with the aim to determine the error of the particle filter with RSSI system setup in comparison to pure RSSI values with characterization. This chapter goes over the thought process of how and why the system was setup in this way, as well as where improvements might be made.

The system setup is presented in section 4.1 which shows the overall flow and relation between the stationary node, mobile node, and base station. It also explains the reasoning and thought process of the system. Sections $4.2,4.3$, and 4.4 goes over the setup process used, if any, before results were gathered.

\subsection{System Setup}

The system can be broken up into two parts: the nodes, and the base station. Waspmotes were used for both the stationary nodes and mobile nodes with XBEE 802.15.4 PRO modules.

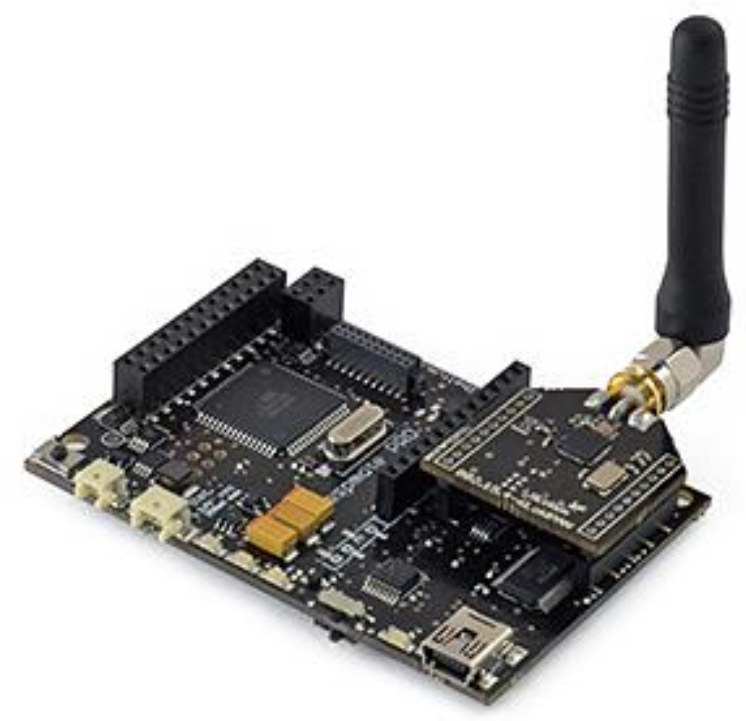

Figure 10: Waspmote with XBEE 802.15.4 PRO module.

The waspmote was chosen because of its specific usage for sensor networks and the abundant libraries available to them due to the Arduino codebase. However, it is important to note for future 
iterations of this thesis for gait analysis applications as well as localization, SHIMMER wearable sensor node would be the best choice for the mobile node. [31] The base station was a simple hp laptop that received information from the mobile node via a USB connection but was fully capable of receiving the packets sent from the mobile node without the use of a wired connection. For the experiments, a direct connection was used.

For the purposes of this thesis, the major processing is done at the base station. It was deemed unnecessary for the mobile nodes to be aware of their location at all times, and was instead allowed the ability to process and adjust the sensors at all times. Because of this, the only relevant information that the stationary node needed to send to the mobile node was its identifier. Power received could be calculated from the packet received. As discussed in the future works section, for the continuation of this thesis, applying an algorithm that allows for stationary node switching would allow for more accurate RSSI estimation readings on a per room basis with each room characterized separately.

Figure 11 shows the diagram of the experimental flow of the nodes and how they interact with each other. The base station is shown in figure 12. Taken as a whole, the experimental design is presented as figures 11 and 12 combined. 


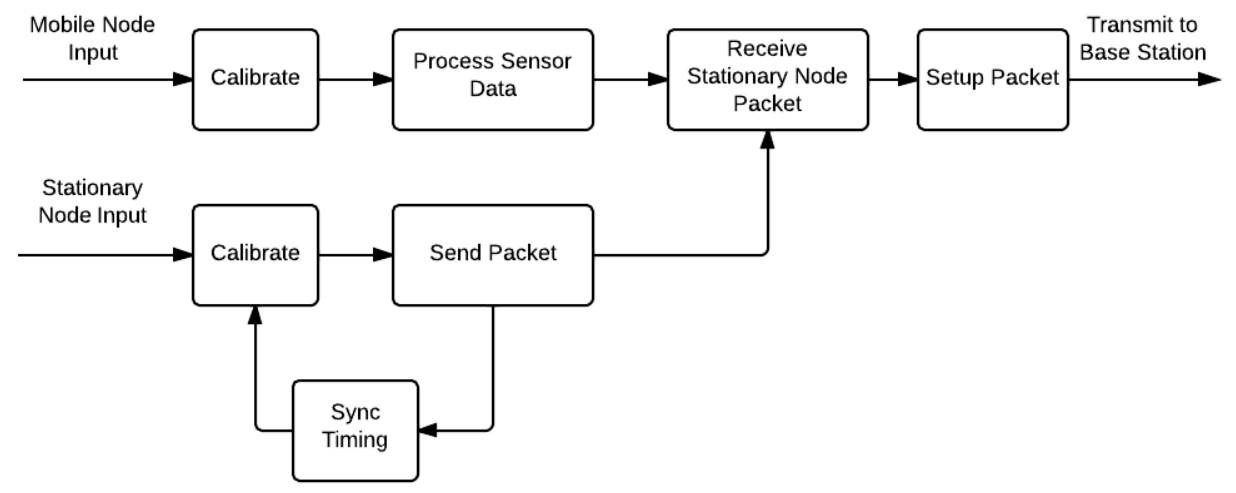

Figure 11: Block diagram of the experimental flow of the sensor hardware, both stationary and mobile and how they interact. Location estimation is not done on the hardware, but instead done at the base station. The sync timing block is used to make sure that information transmitted does not overpower other nodes. This allows for a more consistent delay between packets.

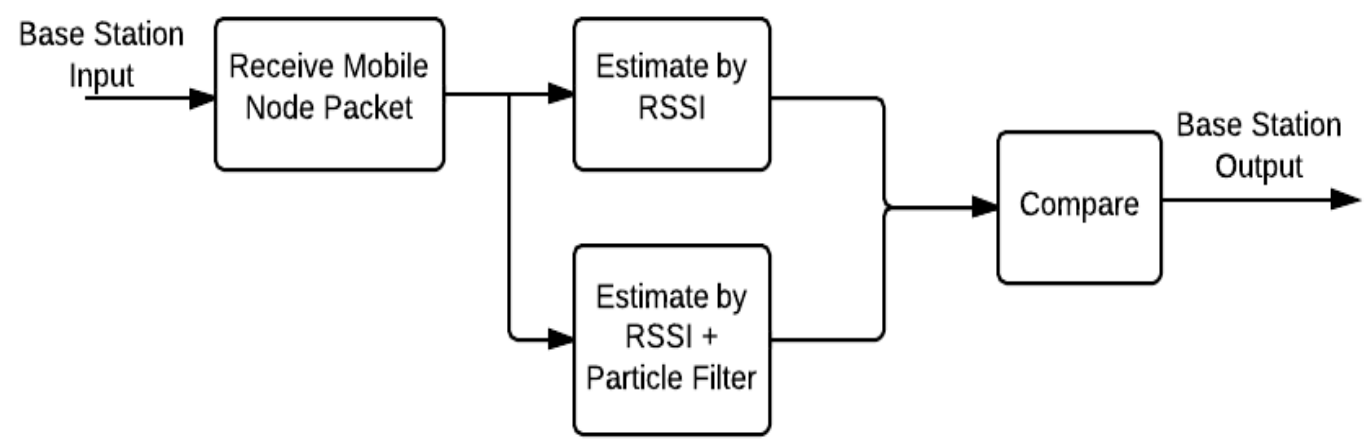

Figure 12: Block diagram of the experimental flow of the base station and the actions the base stations do.

The mobile and stationary nodes, as shown in figure 11, are there only to acquire the necessary information so that the data can be sent off to the base station for processing. The stationary nodes need to calibrate early on to get similar timestamps on each of the nodes so that received packet at the mobile node are within the same timeframe. The sync timing phase is when the root node, stationary node 1 , would send a packet out to all other stationary nodes. Once received, the other stationary nodes would calibrate their timings to the root node's and send their packet at 
a delay. This allowed for consistent packet information to be received at the mobile node without receiving multiple packets from the same node. Figure 11 shows how the processing at the base station is done. The received mobile node packet includes the node power received and the accelerometer and gyroscope readings. The estimate RSSI and estimate RSSI + Particle Filter blocks are compared after they have been calculated to show the actual vs estimates.

\subsection{Sensor Fusion}

The accelerometer used was the integrated Waspmote three axis $(x, y, z)$ accelerometer that can take 2560 measurements per second from $-6 \mathrm{~g}$ to +6 [32]. The gyroscope used was the ITG 3200 three axis breakout board, shown in figure 12 .

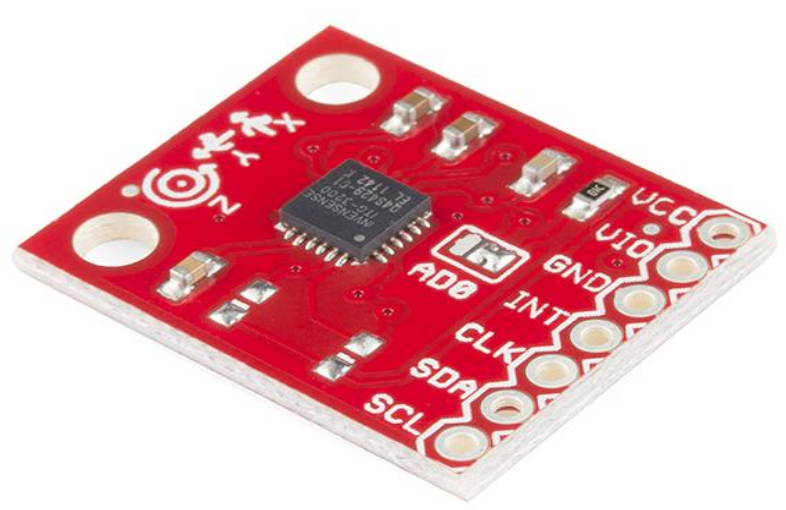

Figure 13: ITG-3200 breakout board [33].

As shown in figure 10, in the calibrate block, the biasing of the system is done at the node. The accelerometer and gyroscope are then used in conjunction with the particle filter to calculate current position as well as create a more stable gyroscopic drift measurement. 
As shown and explained previously in chapter 2, figure 4, the gyroscope and accelerometer are used in conjunction. This creates a complimentary filter for drift correction that is used with the particle filter.

4.3 RSSI Setup

The characterization step, as discussed in chapter 3.1 .3 was done in the $3 \mathrm{~m} \times 4 \mathrm{~m}$ room.

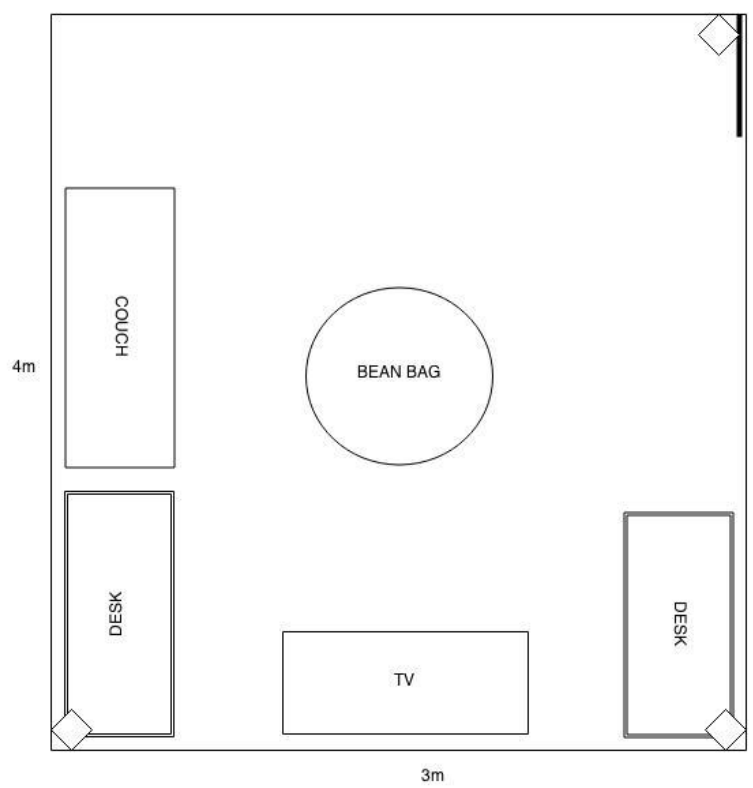

Figure 14: The layout of the $3 \mathrm{~m} \times 4 \mathrm{~m}$ room. Nodes located at the corners.

Table 1 shows the values of the characterization. This setup is required before any actual experimentation can be done as it provides the necessary information to account for obstacles within the room. Each node was placed in the location they would be transmitting from, and then the mobile node was moved to the specified distance. 
Table 1: Environmental characterization step between stationary nodes and mobile nodes with set distance measurements.

\begin{tabular}{|c|c|c|c|c|c|c|c|}
\hline $\begin{array}{c}\text { Node } \\
\text { Name }\end{array}$ & $\begin{array}{c}1 \mathrm{~m} . \\
\text { distance } \\
(\mathrm{dBm})\end{array}$ & $\begin{array}{c}3 \mathrm{~m} . \\
\text { distance } \\
(\mathrm{dBm})\end{array}$ & $\begin{array}{c}3 \mathrm{~m} . \\
\text { distance } \\
\text { obstructed } \\
(\mathrm{dBm})\end{array}$ & $\begin{array}{c}\text { Pathloss } \\
\text { Exponent }\end{array}$ & $\begin{array}{c}\text { Pathloss } \\
\text { Exponent } \\
\text { Obstructed }\end{array}$ & Position & Position \\
\hline $\begin{array}{c}\text { Node 1 } \\
\text { (root) }\end{array}$ & -35 & -45 & -55 & 2.41 & 4.192 & $3 \mathrm{~m}$ & $4 \mathrm{~m}$ \\
\hline Node 2 & -36 & -43 & -53 & 2.41 & 3.563 & $3 \mathrm{~m}$ & $0 \mathrm{~m}$ \\
\hline Node 3 & -36 & -43 & -52 & 2.41 & 3.353 & $0 \mathrm{~m}$ & $0 \mathrm{~m}$ \\
\hline
\end{tabular}

Table 1's node name specifies which stationary node is being recorded as it talks to the mobile node. The one meter distances and three meter distances $\mathrm{dBm}$ value are calculated, and equation 3.8 from section 3.1.3 is used to calculate the environmental characterization using both one meter and three meters as $P_{r(d))}$ and $d_{0}$ and $P_{r(d)}$ and $d$ respectively.

It is important to note that the environmental characterization will change based on the amount of obstacles within any given room. It was seen that with a person standing in front of any given stationary node, the $\mathrm{dBm}$ decreased, on average, approximately $-10 \mathrm{dBm}$. To see the effects of pathloss on this, and to use in the experiment, the pathless exponent obstructed was calculated with the initial $1 \mathrm{~m} . \mathrm{P}_{\mathrm{r}(\mathrm{d} 0)}$ and $\mathrm{d}_{0}$ value.

\subsection{Particle Filter}

The particle filter in this thesis is used to estimate the value of current position based on observable data, such as acceleration and orientation. Also, by using previous and current position data, it is possible to come up with an accurate estimation as to where a target may be in relation to the previous sample $\mathrm{k}-1$. The particle filter is used after RSSI information acquisition and distance conversion step as well as when the accelerometer and gyroscope information are received as well.

Since the coordinates of $X$ and $Y$ in this thesis are considered independent variables, where an independent variable states that the expected value of $X$ has no effect on the expected value of $Y$. Because it can be said that $E(X Y)=E(X) E(Y)$ we can simplify our particle filter by isolating $X$ 
and $Y$ by themselves and thus reducing the complexity of the model that is necessary for modeling our system to get accurate results from our particle filter. 


\subsubsection{Particle Filter Characterization}

In order to test the particle filter before applying it with RSSI information, for the purpose of this thesis, the random walk model was used to adjust and characterize how noise from a sensor affects the output of the particle filter itself. Noise, in relation to the particle filter, comes from two different sources. The system (the observed values) and the sensors (the noise from the sensors themselves when taking data). The observed noise is a value that is tweaked and cannot be physically measured in the system itself. The sensor noise is the noise that can be characterized and modeled as some form of Gaussian white noise or other type of noise. As seen from figures 15,16 and 17, the noise of the measurement was changed, while the noise of the system remained the same. The number of particles used for these calculations was 1000 . The below code snippet is the meat of the particle filter characterization and is what is used to generate Figures $14-18$.

\#Update the particle filter $x$ out and $z$ obs

PF. $x=\left(0.5^{\star} \mathrm{PF} . \mathrm{x}+25^{\star} \mathrm{PF} . \mathrm{x}\right) /\left(1+\mathrm{PF} . \mathrm{x}^{\star \star} 2\right)+8^{*} \cos \left(1.2^{\star}(\mathrm{t}-1)\right)+\mathrm{PF} . g$ en_system_noise () ;

$z=P F . x^{* *} 2 / 20+P F . g e n \_m e a s u r e \_n o i s e() ;$

\#Update the weights of the particle filter. for $i$ in range(PF.N):

x_P_update $[i]=\left(0.5^{\star} P F . x \_P a r t i c l e s[i]+25^{\star} P F . x \_P a r t i c l e s[i]\right) /\left(1+P F . x \_P a r t i c l e s[i]^{\star \star} 2\right)+8^{*} \cos \left(1.2^{\star}(t-1)\right)+$ PF.gen_system_noise();

z_update[i] $=\left(\right.$ x_P_update $\left.[i]^{* \star 2}\right) / 20$;

P_w $w i]=\left(1 / \operatorname{sqrt}\left(2^{*} \mathrm{pi}^{*} \mathrm{cov}\right)\right){ }^{*} \exp \left(-\left(\mathrm{z}-\mathrm{z}_{-} \text {update }[\mathrm{i}]\right)^{\star *} 2 /\left(2^{*} \mathrm{cov}\right)\right)$;

$P \_w=n p . t r u e \_d i v i d e\left(P \_w, n p . s u m\left(P \_w\right)\right)$

\#Update particles

for $\mathrm{i}$ in range(PF.N):

vals $=n p$. where $\left(n p\right.$. random.uniform ()$\left.<=n p . \operatorname{cumsum}\left(P \_w\right)\right)$

PF.x_Particles[i] = x_P_update[vals[0][0]];

The noise is applied at the observation (z) and the output (PF.x) for their respective parts. 


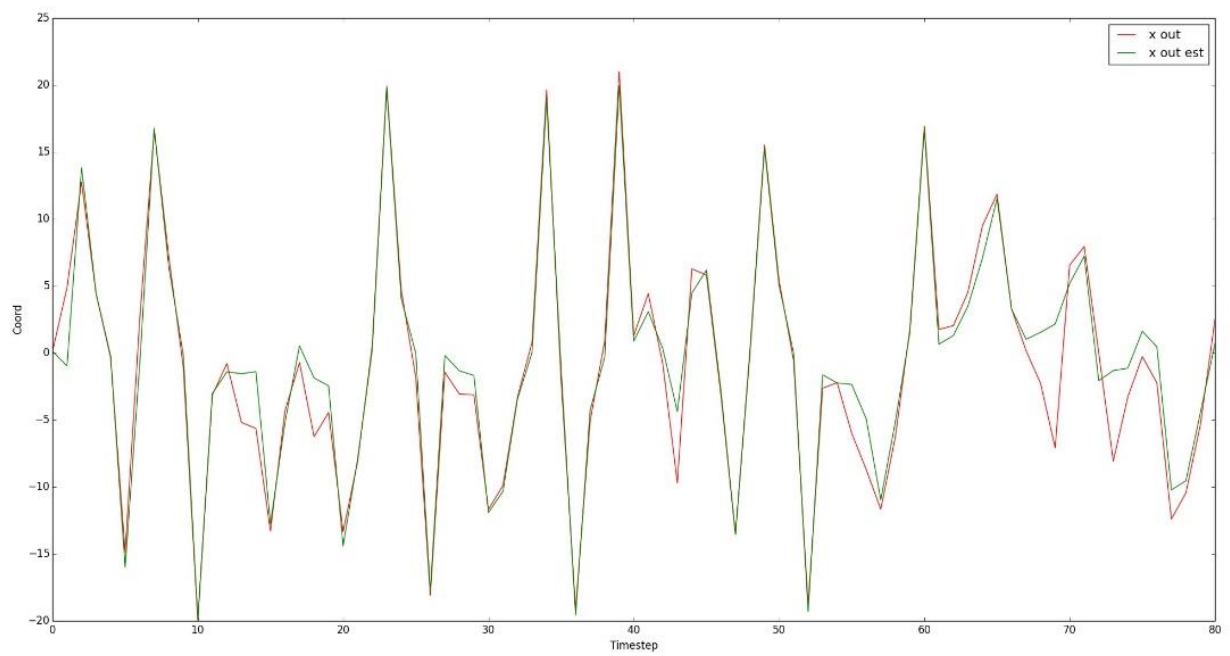

Figure 15: Measurement Noise with mean of 0 and sigma of 1, System Noise with mean of 0 and sigma of 1

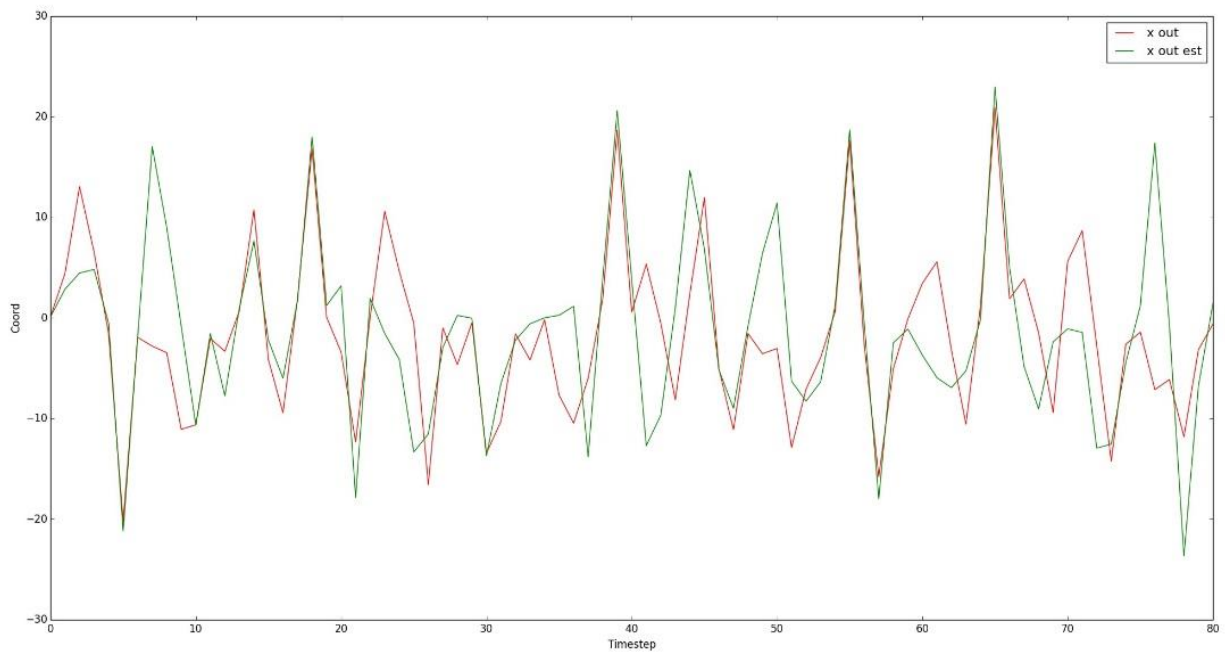

Figure 16: Measurement Noise with mean of 0 and sigma of 5, System Noise with mean of 0 and sigma of 1 


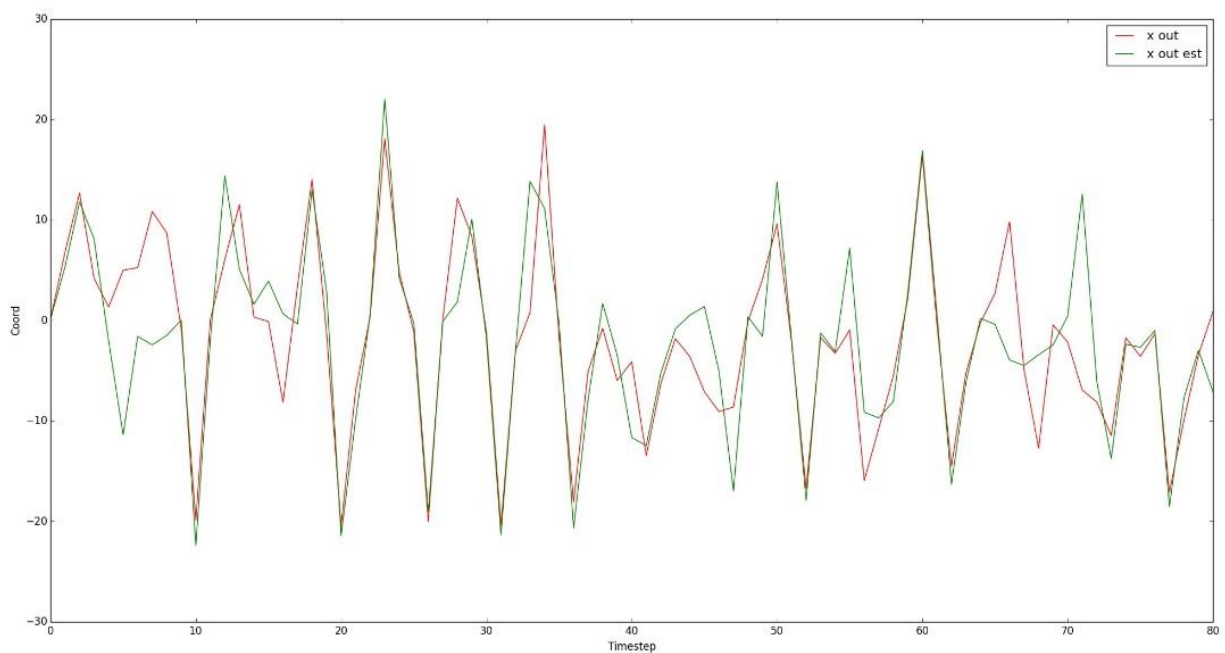

Figure 17: Measurement Noise with mean of 0 and sigma of 10 , System Noise with mean of 0 and sigma of 1

As seen from figures 15,16 , and 17 with an increase in sigma, which is the square root of the variance, the outputted estimate itself becomes less close to the approximation, but within acceptable parameters. This is expected of the characteristic of the noise. With a decrease in the noise of the sensors, a more smooth representation that adhered to the actual value occurred. This shows that for higher grade sensors with lower noise values, it can increase the accuracy of the system. This information can be directly correlated to the sensor fusion of RSSI and INS. The more accurate the observed value (INS) is to the system value (RSSI), the smoother the output.

Next, we take the system noise while holding the measurement noise constant to see how it affects the particle filter. 


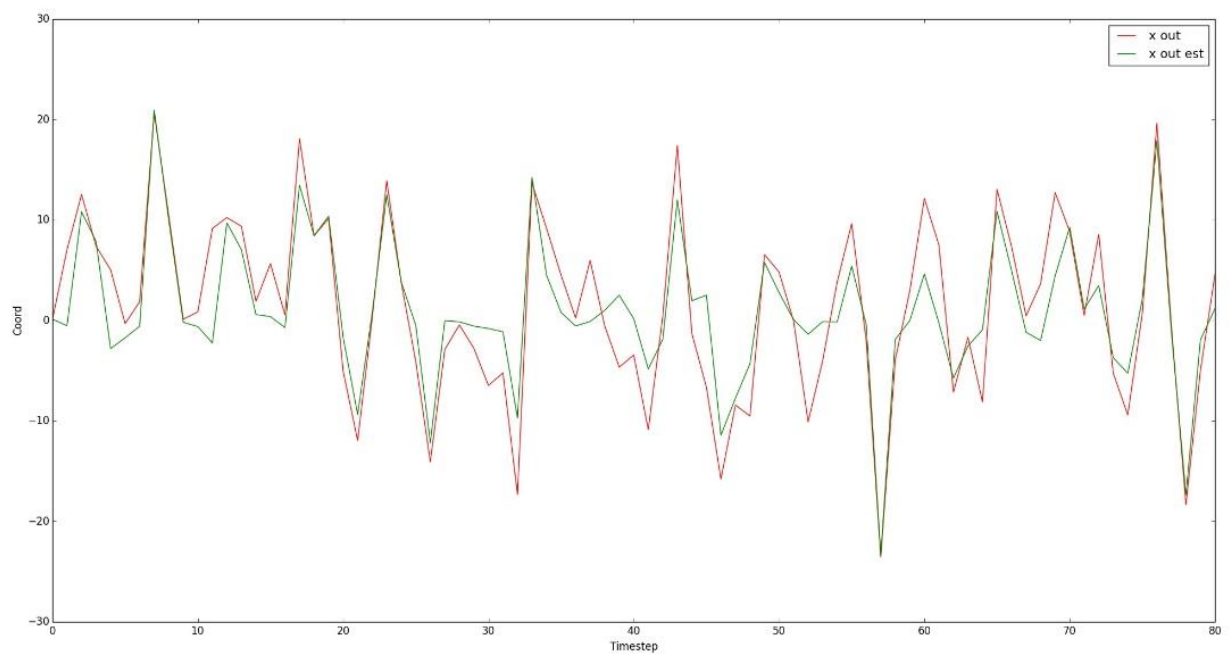

Figure 18: System Noise with mean 0 and sigma of 5 , Measurement Noise with mean 0 and sigma of 1

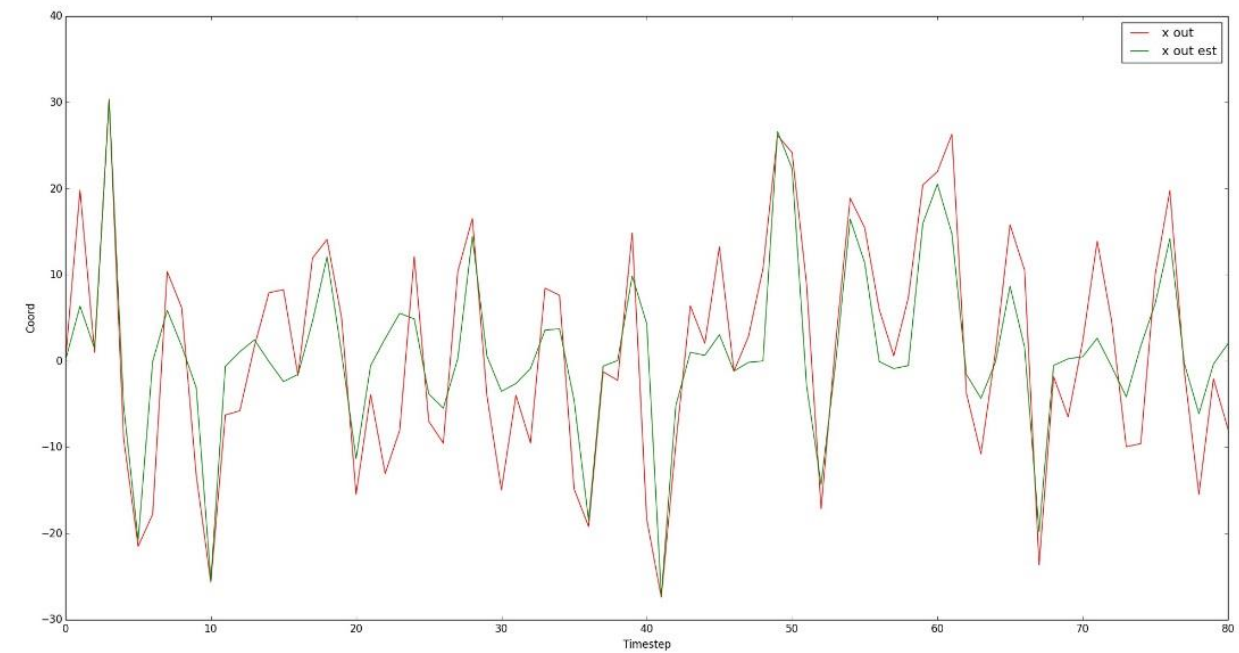

Figure 19: System Noise with mean 0 and sigma of 10, Measurement Noise with mean 0 and sigma of 1

With figures 15, 18, and 19, the noise of the system was adjusted. By adjusting the observable noise, it has a much greater impact on the particle filter's ability to determine a proper estimate of the output. This means that a more noisy system value (RSSI) has a bigger impact than noisy sensors. This means, for the biggest correction in particle filter output, having an accurate system 
value gives the most correct particle filter estimate. However, it is important to note that the particle filter still gives a smoothed out accurate representation of the actual that it believes the system is at. This means, that regardless of the validity of the two sensor values, as long as they are in some way correlated and can have a measurable 'correctness' to their outputs, the particle filter will give a fused value of the sensors themselves. 


\section{CHAPTER 5 RESULTS AND ANALYSIS}

In this section, the particle filter output data in the $X$ and $Y$ axis is compared with the INS and RSSI only estimations in a $3 \mathrm{~m} \times 4 \mathrm{~m}$ room. The RSSI algorithm from [24] is explored and improvements are made to increase accuracy using RSSI localization through the use of assumptions and extra characterizations, which in turn improved the sensor fusion of the RSSI and Inertial Navigation System (INS). INS errors are explored to give the reader an understanding of issues associated with using it, and a solution to the problems are presented.

We also explore various domain specific assumptions for the particle filter through the demonstrations of tests that occurred and their findings. 


\subsection{INS and RSSI Positional Error Correction}
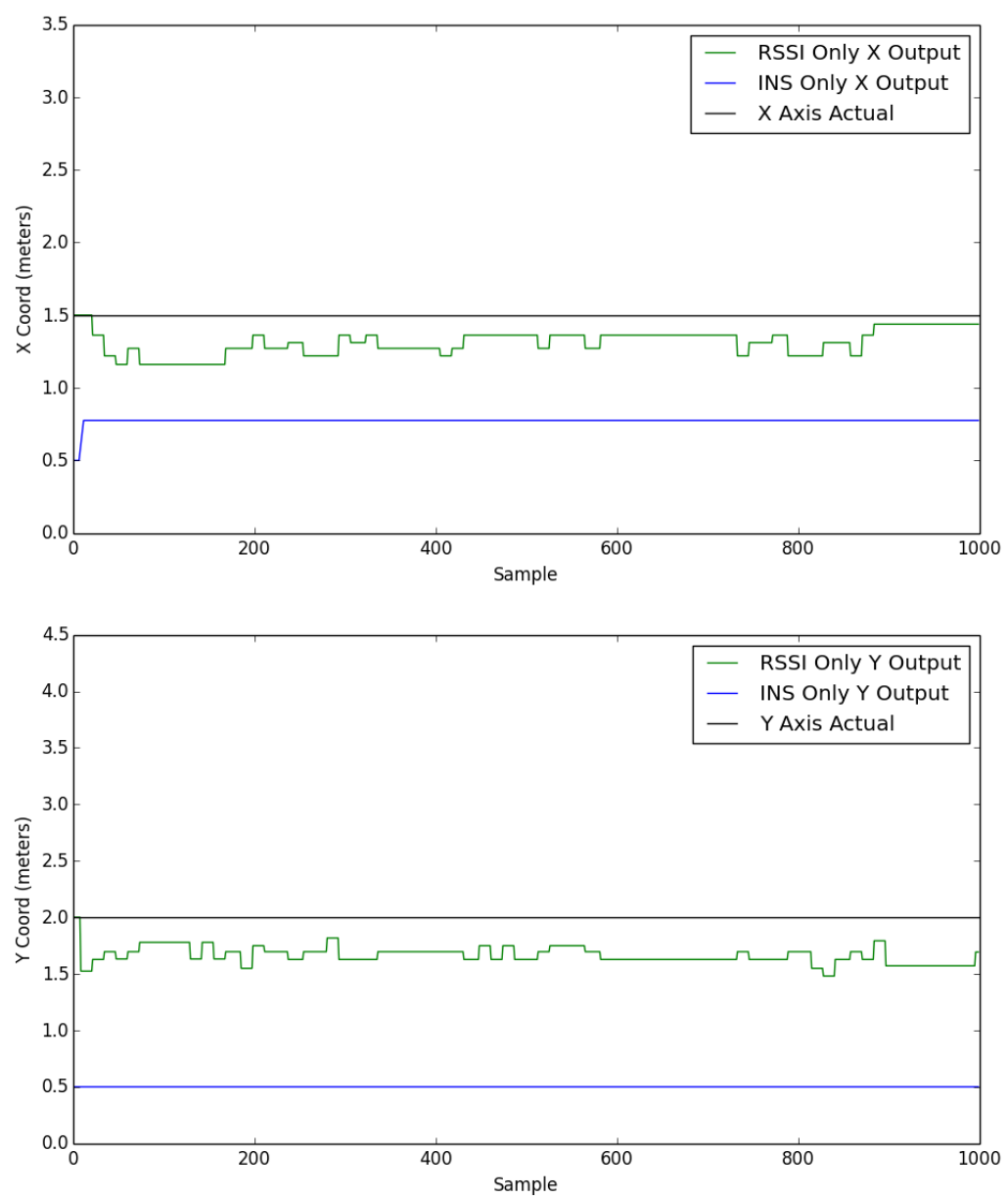

Figure 20: Stationary Poor INS Starting condition with actual node position at $(1.5 \mathrm{~m}, 2.0 \mathrm{~m})$. Improper calibration of the INS causes a biased positional error, as shown in figure 20.

The actual node position was located at $(1.5 \mathrm{~m}, 2.0 \mathrm{~m})$ and while the INS output remained steady in both the $X$ and $Y$ coordinates, error between the actual coordinates and INS estimation was a positional value of $1.5 \mathrm{~m}$ in the $\mathrm{Y}$ axis and $.7 \mathrm{~m}$ in the $\mathrm{X}$ axis, or $75 \%$ and $46.6 \%$ respectively. $\mathrm{RSSI}$ information provides a consistent form of position bias correction with an error of approximately $.4 \mathrm{~m}$ at its greatest, or $20 \%$. 

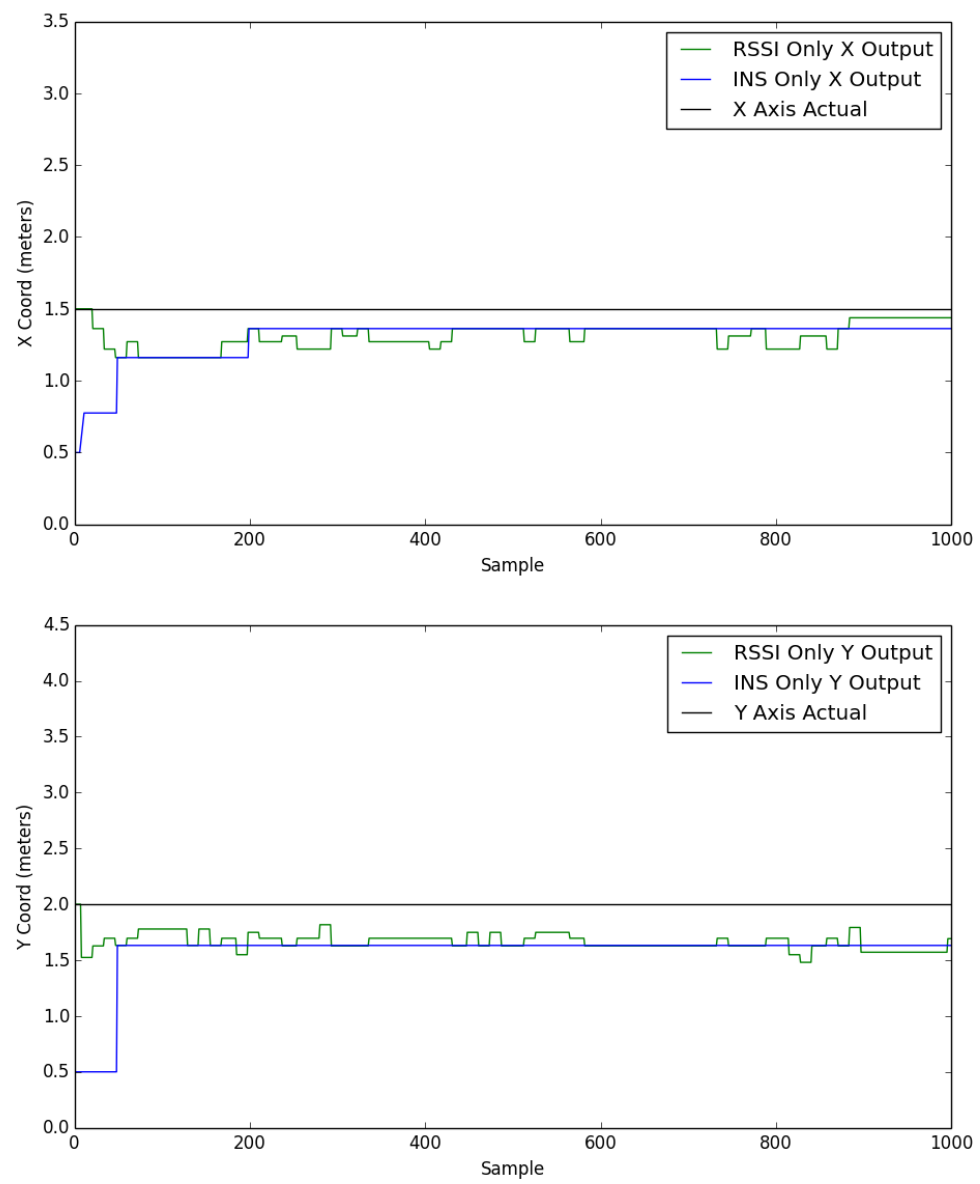

Figure 21: Corrected Stationary Poor INS Starting condition with actual node position at $(1.5 \mathrm{~m}$, $2.0 \mathrm{~m})$.

Due to the noise of the RSSI value, INS information is only updated if, while stationary, the observed value of the position deviates by more than .2 meters in the $\mathrm{x}$ or $\mathrm{y}$ direction As such, INS error is reduced to .2 in the $x$-axis and .4 in the $y$-axis, which is a $13.3 \%$ and $20 \%$ error as seen in figure 21.

It was assumed that in order for a person to be stationary, the inertial measurement unit (IMU), would need to be unchanging for at least five seconds. This is to avoid inadvertent positional corrections that could lead to larger errors due to RSSI errors during movement. It is 
believed that five seconds provides enough time for the INS algorithm to update itself such that there is no accidental positional updates on the IMU. RSSI errors will be discussed later
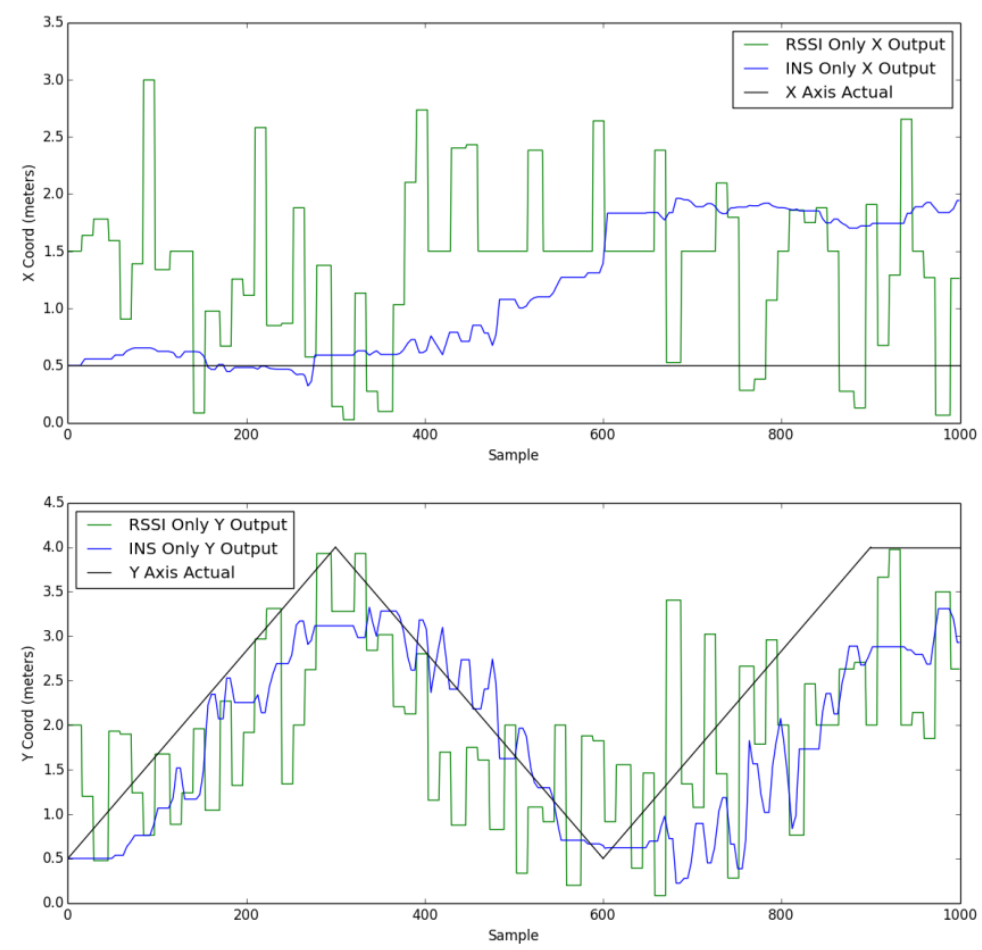

Figure 22: Vertical movement, Y-axis only. Gyroscopic drift in X direction. Without INS positional correction algorithm.

As stated previously in section 1.2, gyroscopic drift is the contributing factor to positional inaccuracies in IMUs. Figure 22 displays this drift in the form of the $x$ coordinates of the IMU drifting from $0.5 \mathrm{~m}$, which is the actual, to $1.5 \mathrm{~m}$ and is an unfixed representation of the INS output. This drift in the gyroscope caused an error in both the INS $x$ and $y$ values which can be caused by all of the previously stated sensor noise errors. To fix this, domain specific assumptions and additional hardware such as magnetometers are used. For positional estimations that could be used to correct for gyroscopic drift, angle of arrival (AoA) or time difference of arrival (TDoA) could be used as shown in [18]. By using TDoA over magnetometers for drift correction, the approach was able to remove more than $85 \%$ of the inserted drift in the gyroscope signal [18]. 
For localization, the TDoA algorithm can therefore be used two fold to both gain positional estimation data as well as provide a method of gyroscopic drift correction as it as the leading cause of position error in the INS.

RSSI positional output for estimation can be seen in figure 22. Due to the fact that the signal attenuation is proportional to distance between the stationary and mobile nodes, and the fact that the space is constrained, the maximum attenuation for an unblocked signal can be calculated within the constrained space. Thus, when you observe attenuation greater than the value specified by the obstructed $3 \mathrm{~m}$. $\mathrm{dBm}$ value in table 1 , located in section 4.3 , it can be inferred that there is an obstruction. Once this has been determined, you can apply the pathloss $_{\text {obstructed }}$ instead of the pathloss unobstructed $_{\text {to }}$ the RSSI trilateration calculations to get a better estimate of the position of the node.
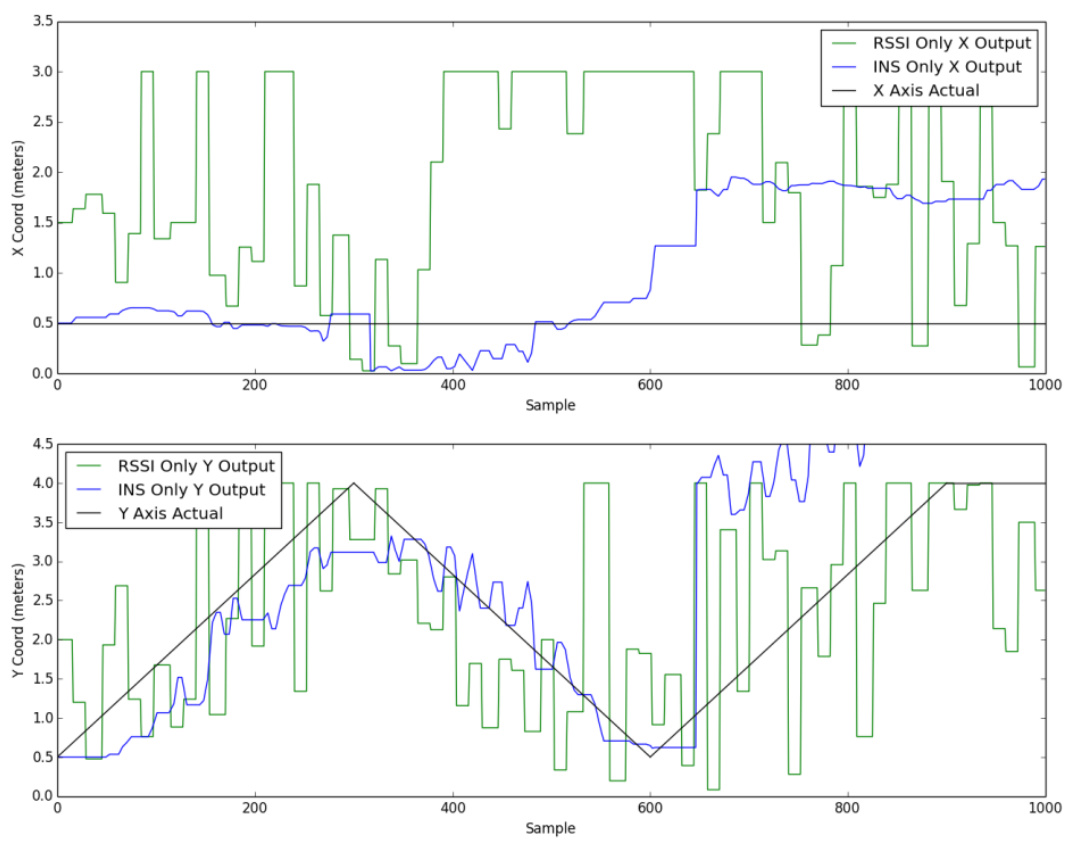

Figure 23: Vertical movement, Y-axis only without algorithm corrections for RSSI and with INS algorithm corrections. 
Figure 23 shows an uncorrected RSSI output with INS output using the previously stated position bias fix where if the INS believes itself stationary, it will correct for its positional drift by using the RSSI's value. This is shown at 650 samples. It is also important to note that to correct for multipath upfade, any positional signal larger than the size of the room is divided in half since $\mathrm{S}_{1}+\mathrm{S}_{2}=\mathrm{RSSI}_{\text {out }}$, where RSSI out should only include $\mathrm{S}_{1}$. Multipath nulls are uncorrected for as values of 0 are valid and would be corrected for by setting $\mathrm{RSSI}_{\text {out }}$ to the mean of the current RSSI output which would average out consistently at the midpoint of the room.
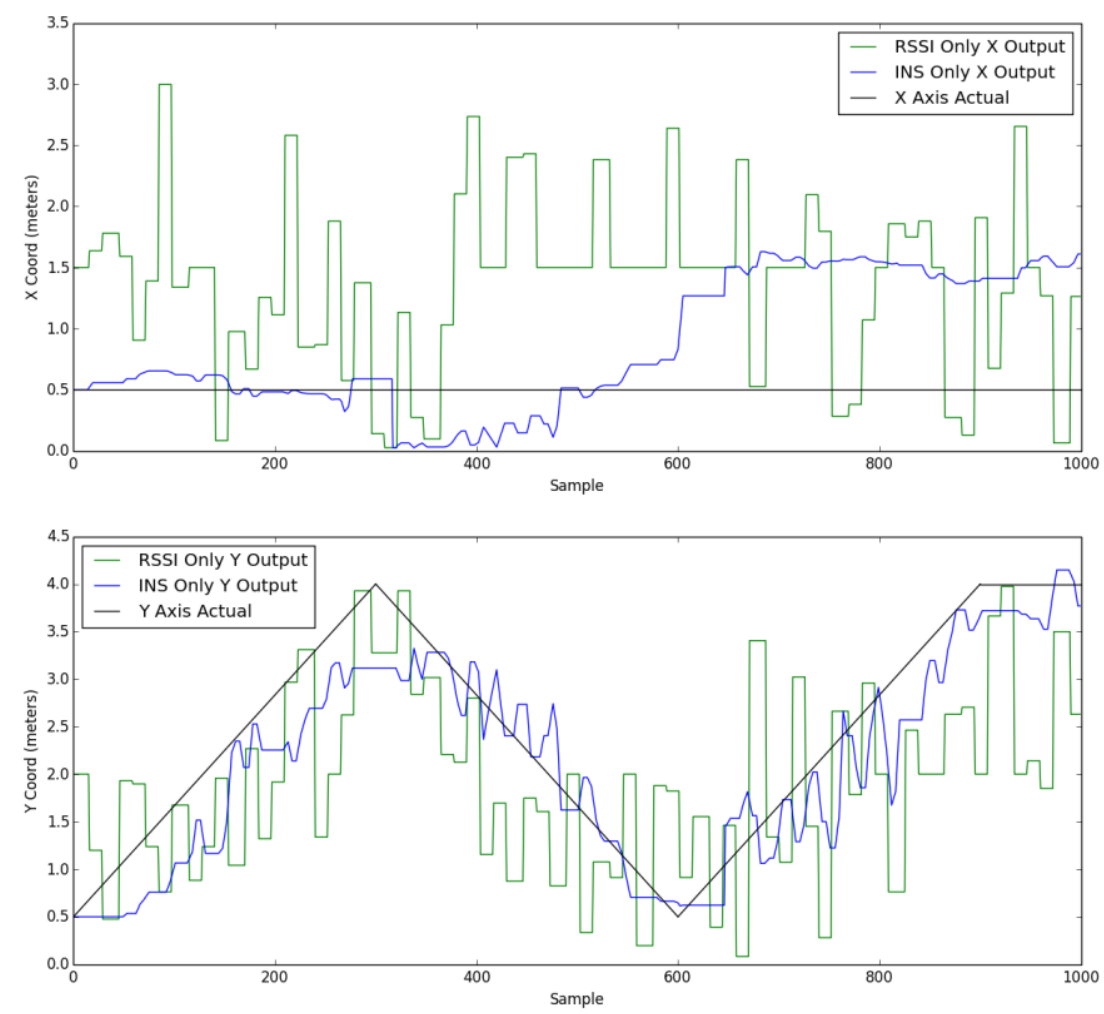

Figure 24: Vertical movement, Y-axis only with algorithm corrections for RSSI and INS

Finally, figure 24 shows the RSSI and INS corrections together. It can be seen that the INS output has an approximate $25 \%$ positional error at maximum in the y direction at 300 
samples, and $200 \%$ positional error in the $x$ direction at maximum as opposed to the positional error in figure 21 where INS positional drift correction was unused. 


\subsection{Sensor Fusion - INS and RSSI}
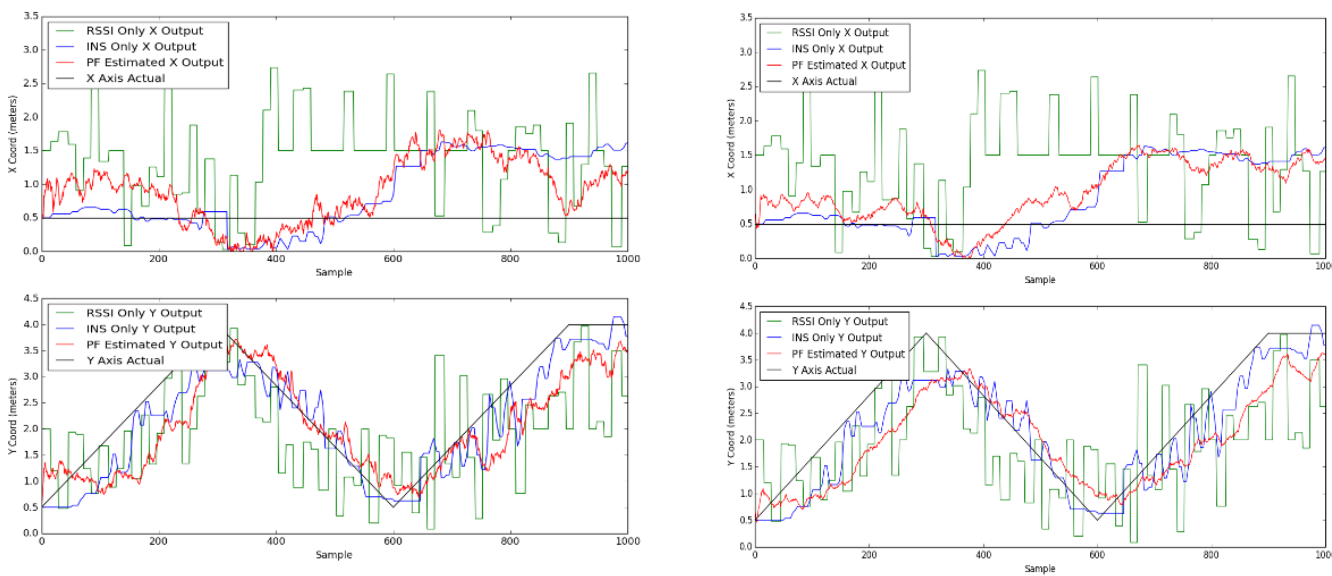

Figure 25: Sensor Fusion - Particle Filter - INS and RSSI combined, Y-axis movement, 100 particles (left), 500 particles (right)

Figure 25 is the same data with 100 particles (left) and 500 particles (right) used in the particle filter. While the two figures follow a similar path, the output of the particle filter is smoothed out with the higher number of particles. There is no specific way to tell how many particles should be used for calculations, but for the purposes of this thesis, it was deemed 500 particles to be a sufficient amount. This is due to the smoother nature of the signal between to the two positional outputs of RSSI and INS that it was fusing when using 500 particles as seen in figure 26. Also, 100 particles had more variance in its output due to the RSSI value it was fusing, which was one of the things this thesis was trying to avoid. 

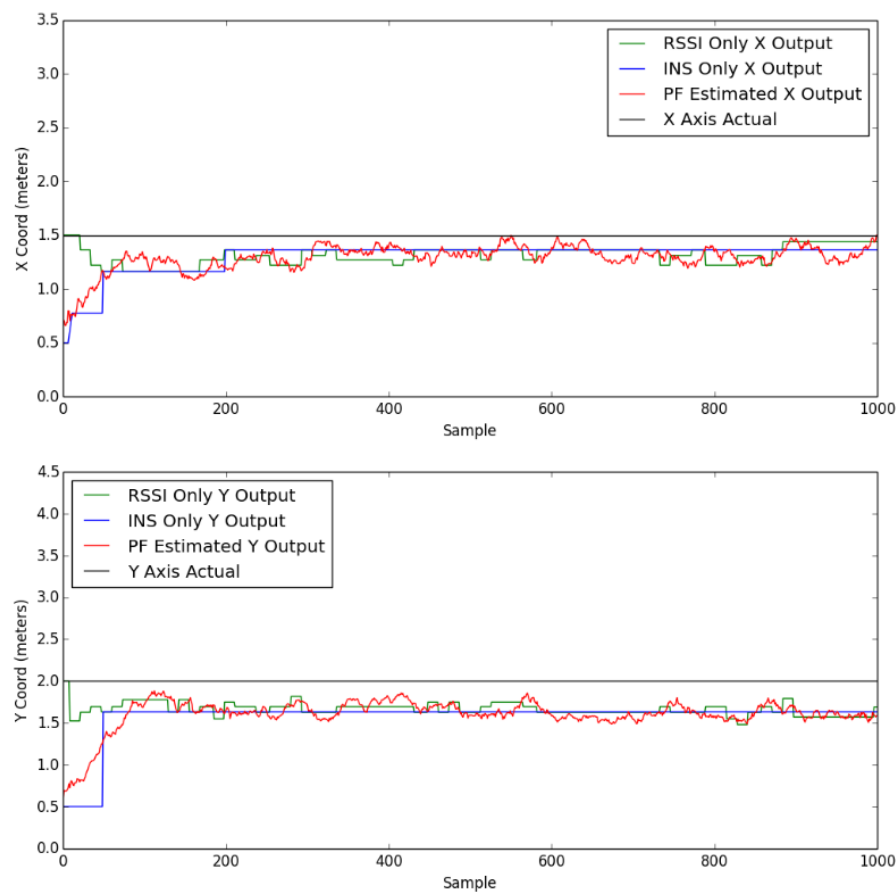

Figure 26: Sensor Fusion - Particle Filter - INS and RSSI combined, Stationary, 500 particles

As stated previously, sensor fusion is the combination of two or more signals. Through the error corrections done for both the RSSI and INS outputs, each signal has been fixed to be as accurate as possible at any given time with justification for changes documented in 6.1.

Since the particle filter relies heavily on the output of two or more related systems and the accuracy of their signals, any error that is associated with either of the signals themselves will cause an error in the output of the particle filter. The job of the particle filter for sensor fusion is to smooth the information of the sensors such that it is the most likely estimate of the output based on the information given. For this reason, weighting of the INS and RSSI signals have been done in the particle filter due to the inherent inaccuracies of RSSI. While hard to put into distance errors and other such concepts, figure 27 shows the error of RSSI and INS, which allows for us to deduce which signals are a more accurate representation of the node and its position value, and therefore, which signal would give us the more accurate output of the particle filter by weighting it 
higher and essentially stating, 'this value is believed to be a more accurate representation of our actual position value.'.

Figure 27 shows the particle filter output in comparison to the RSSI and INS outputs. It follows the INS value as it is believed to be the more accurate representation of the positional estimation, but is lifted up towards the proper actual value at zero to 75 samples due to the RSSI. From there, the particle filter shifts with RSSI outputs.
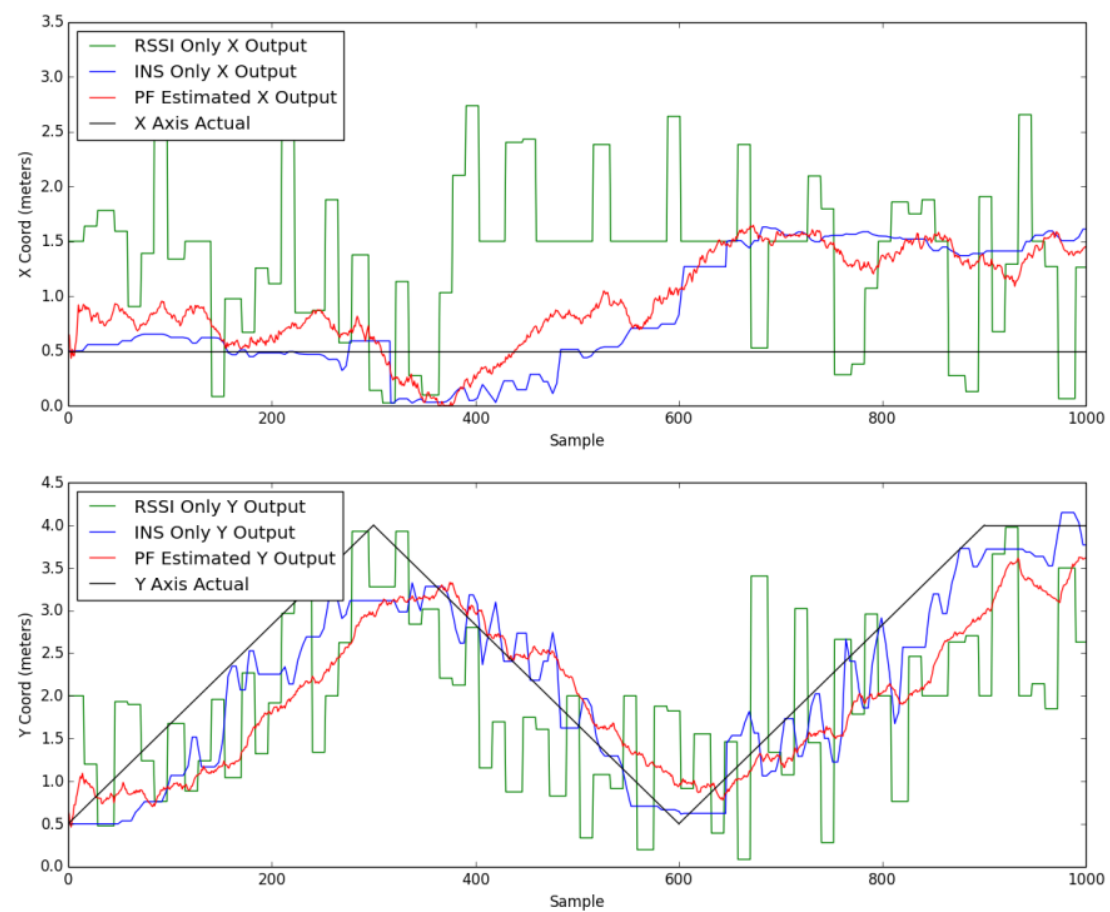

Figure 27: Sensor Fusion - Particle Filter - INS and RSSI combined, Y-axis movement, 500 particles 

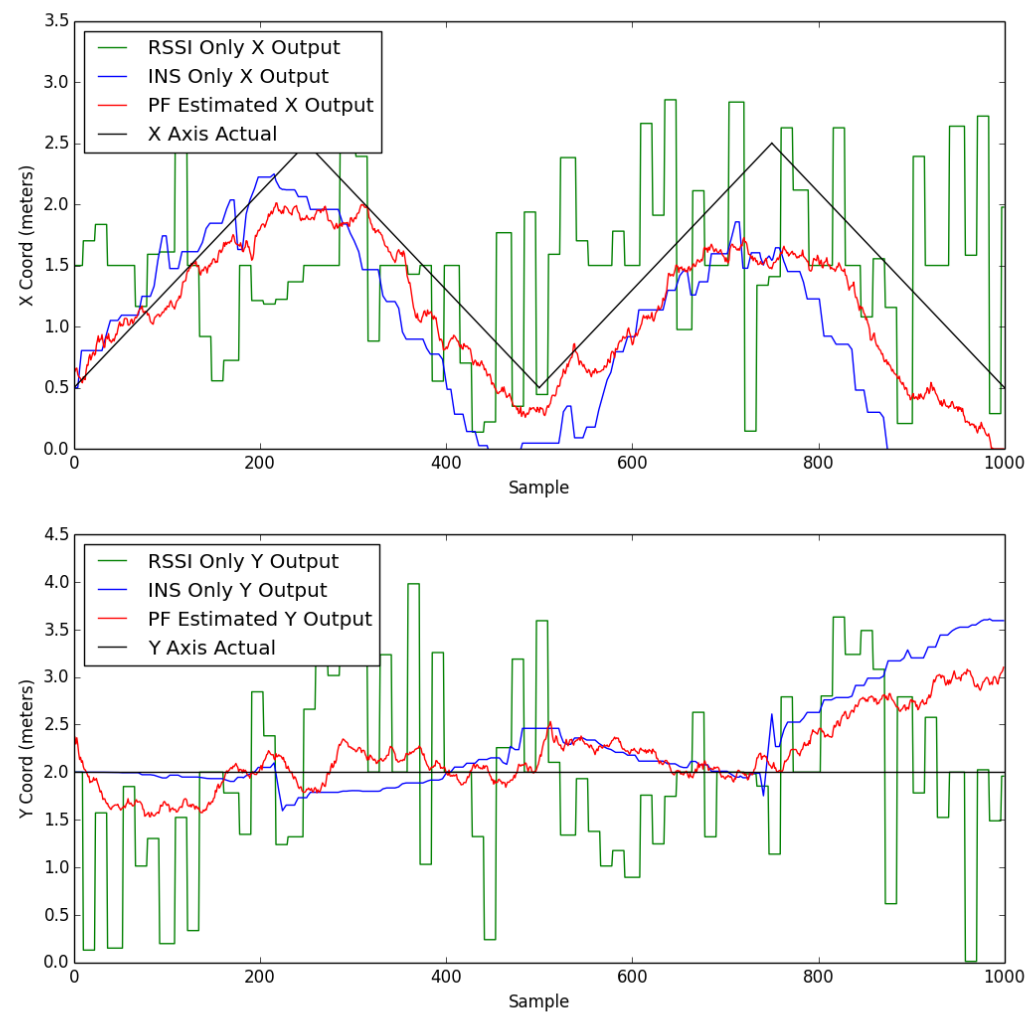

Figure 28: Sensor Fusion - Particle Filter - INS and RSSI combined, X-axis movement

Figures 27 and 28 show the particle filter movement for the output when walking in a horizontal and vertical manner. In figure 27, while the particle filter isn't the closest approximation for the actual position of the node itself, it is a more smooth and noiseless output, with little deviation from where it believes the position of the node to actually be. In figure 27 , with the INS and PF output, the $x$ coordinates vary between 0.0 and 2.0. It is important to note that while the particle filter does not give a more accurate representation of the actual output for either the $\mathrm{X}$ or $\mathrm{Y}$ coordinates, it does not give a less accurate output either.

As stated previously, the particle filter output follows the INS value as it believes the value to be the closer approximation of the correct output, but the particle filter also takes into consideration the output of the RSSI values. Since the RSSI values were also lowering and rising, much like the INS values, the particle filter believed that the true value of the $x$ coordinate was 
actually increasing due to the assumed correlation between the RSSI and INS positional estimations. This correlation is such that, when RSSI increases, and INS increases, it must be that the true output is also increasing.

From figure 28 , it can be seen that the particle filter follows the actual more closely than any of the other two signals. This is due to the RSSI output stating that the actual was non-zero, even though the INS was stating otherwise. At sample slice 850 of figure 28 , it can be seen the actual value was approximately $1.75 \mathrm{~m}$ at the time, whereas the INS value is approximately $.75 \mathrm{~m}$ and the particle filter is $1.5 \mathrm{~m}$. It can also be seen, from figure 27 the $X$ and $Y$ axis actual lines are closely followed by the particle filter in both cases. 


\section{CHAPTER 6 DISCUSSION AND FUTURE WORKS}

\subsection{Discussion}

The proposed system saw an increase in accuracy in comparison to the RSSI only and INS only based systems for room specific localization. From the results in 6.2, the major contributing factor to particle filter inaccuracies came from the inaccuracies in position of RSSI and INS. RSSI inaccuracies came from dBm calculations and conversion from multipath sources, and INS inaccuracies came from gyroscope drift that caused positional changes in $\mathrm{X}$ and $\mathrm{Y}$ axes. The difference of this can be seen in subplot 1 of figure 21 where the actual positional estimate was constant for the $\mathrm{x}$-coordinate, but positional changes were still registered for the $\mathrm{x}$ axis due to the gyroscopic drift.

While the particle filter did provide more accurate results for positional data, the positional estimates that were used in the particle filter were inaccurate, or were not full compensated for their errors.

Correction for gyroscopic drift and positional errors of RSSI could be corrected by using TDoA or AoA localization techniques to correct for both gyroscope drift and give a better representation of the actual position. By using an algorithm which is less prone to multi-path and

more accurate as a system state variable, the accuracy of the system would increase with a particle filter. This is why GPS/INS is often times used with an Extended Kalman Filter, as it provides additional values to give the true positional estimates.

Reduction in the multipath error could be reduced by looking at various techniques such as MIMO, OFDM, Guard Band Intervals, interleaving, and equalization techniques.

Localization is still a topic that should be further researched and discussed. Especially as smaller sensors and stronger microprocessors come out each year for better computation power and sensing. Using RSSI and INS in conjunction together did give more accurate positional results, but I would recommend using a better localization technique other than RSSI. While it is the easiest to implement, it is highly inaccurate. 


\subsection{Future Works}

For the continuation of this thesis, the following steps can be taken to further the discussion of indoor localization tracking for the current system. These topics would create a considerable increase in efficiency and accuracy:

- Convert 2D coordinate system to 3D coordinate system so that height can be considered in the position estimate.

- Add additional hardware to further correct the inaccuracies of position estimation with RSSI without compromising the size or life of the system. Things like adding TDoA, ToA, $\mathrm{AoA}$, and other ranging techniques to the system to compliment the RSSI ranging that is inherent would allow for a stronger estimation in position for the particle filter, and therefore reduce inaccuracies that occur from a noisy and unstable system.

- Use newer generation sensors, as the use of noisy and unstable biased sensors degrade filter performance. This degradation can be seen in the results of the particle filter simulation when comparing the results of filter performance in a system with more noise and a system with less noise. Further investigations into noise related performance issues should be taken into consideration for future iterations of this work.

- Lowering sampling rates degrades the performance of the filter by introducing more inaccuracies into the filter itself. As real world systems will likely reduce the sampling rate due to energy consumption and battery life considerations, Investigation into how sampling rate affect the system and how this can be solved should be considered

- Applying a magnetometer to the system would allow for bias correction for the other sensors that have already been put on the system.

- The current filter used is the particle filter, or bootstrap filter. Other iterations of the particle filter, such as the unscented particle filter, might provide a more accurate filter for the purpose of this system. 
While the above is applicable to the current iteration of the system, there are several things that must be addressed before it can be considered usable for indoor medical applications.

- WSN Protocol Implementation: While the current system is a simple wireless sensor network in its own way, implementing a network algorithm such as LEACH-M for the network protocol will allow for a longer lasting system. This would allow nodes much further away from the base station to maintain a steady battery life and thus average out the lifespan of the network for ease of maintenance.

- Stationary Nodes Switching: In a system where the WSN is distributed across multiple rooms, and with the RSSI algorithm itself, the stationary nodes are assumed stationary. However, movement from room to room does not account for the switching that might need to be facilitated between the stationary nodes such that the RSSI value in each room remains as accurate as possible.

- Hardware Integration: Instead of using a development kit like the waspmote, before implementation of the system, it would be wise to create a device for the specific purpose of running this system.

- Multipath Considerations: Multipath can affect the system in a variety of ways. The environmental characterization step as discussed previously is one of several things that need to be taken into consideration when attempting to get accurate RSSI measurements. Possible solutions include leaving the stock XBEE protocol and creating a new protocol specifically to handle multipath considerations.

- Pulses vs. Continuous Transmission: Tied in with the WSN Implementation, by doing pulse transmission at quick timesteps rather than a continuous transmission, it would be possible to extend the duration of life of all nodes involved. This comes with drawbacks of possibly increasing the error of RSSI as it is no longer instantaneous data, but rather discrete.

- Dynamic RSSI Characterization: Algorithmically come up with a way to increase the environmental characterization of the system based on the amount of mobile nodes 
currently in the area. By doing so, this can account for more obstacles, people, being added to the room. Thus increasing accuracy. This is also necessary for when furniture is moved into and out of the room. Possible solutions to this problem include:

- Using the stationary nodes as the method of rssi characterization within the system. Thus allowing for constant characterization.

- Having a node set in the center of the room that is only used as a control variable for characterization.

- Mobile Node Processing: Processing at the mobile node is currently minimum. By having the mobile node do its own processing and sending that to the base station for comparison may improve the location estimation. 


\section{REFERENCES}

[1] W. Mann, "The aging population and its needs," IEEE Pervasive Computing, vol. 3, no. 2, pp. $12-14,2004$

[2] Redondi, A.; Tagliasacchi, M.; Cesana, M.; Borsani, L.; Tarrio, P.; Salice, F. LAURALocalization and Ubiquitous Monitoring of Patients for Health Care Support. In Proceedings of $21^{\text {st }}$ International Symposium on Personal, Indoor and Mobile Radio Communications Workshops, Istanbul, Turkey, 26-29 September 2010.

[3] B.R.; McGrath M.J.; O'Shea T.J.; Kuris B.; Ayer S,M.; Stroiescu F.; Cionca V. Burns, A.; Greene. Shimmer a wireless sensor platform for noninvasive biomedical research. In Sensors Journal, IEEE, September 2010.

[4] Jawbone Inc. Wireless wristband for a healthier life. http://jawbone.com/up, 2014. [Online; accessed 29-December-2014]

[5] $\mathrm{H}$ Zhou and $\mathrm{H} \mathrm{Hu}$. Human motion tracking for rehabilitation a survey. Biomedical Signal Processing and Control, 3(1):1-18, 2008.

[6] Inertial Navigation. Inertial navigation forty years of evolution. Evolution, 13(3):140-149, 1998.

[7] Oliver J. Woodman, C Oliver J. Woodman, and Oliver K. Woodman. An introduction to inertial navigation, 2007

[8] Eric Foxlin. Chapter 7. Motion tracking requirements and technologies. Environment, pages 163-210, 2002.

[9] HENDERSON, T. C., DEKHIL, M., KESSLER, R., AND GRISS, M. Sensor fusion. In Control Problems in Robotics and Automation (Berlin, 1998), B. Siciliano and K. Valavanis, Eds., Spnnger-Verlag, pp. 193-208.

[10] Luttwak, A. Human Motion Tracking and Orientation Estimation Using Inertial Sensors and RSSI Measurements. M.Sc. Thesis, School of Computer Science, Hebrew University, Jerusalem, Israel, 2011.

[11] Xiaoping Yun and Eric R Bachmann. Design, implementation, and experimental results of a quaternion-based kalman_Iter for human body motion tracking. IEEE Transactions on Robotics, 22(6):1216\{1227, 2006.

[12] Eric Foxlin. Pedestrian tracking with shoe-mounted inertial sensors. IEEE Computer Graphics and Applications, 25(6):38\{46, 2005.

[13] Huiyu Zhou, Huosheng Hu, and A Motivation. Kinematic model aided inertial motion tracking of human upper limb. 2005 IEEE International Conference on Information Acquisition, pages 150\{155, 2005.

[14] Tsenka Stoyanova, Fotis Kerasiotis, Aggeliki Prayati, and George Papadopoulos. Evaluation of impact factors on rss accuracy for localization and tracking applications. Proceedings of the 5th ACM international workshop on Mobility management and wireless access MobiWac 07, $\operatorname{Im(17):9,~} 2007$.

[15] Hui Liu, Houshang Darabi, Pat Banerjee, and Jing Liu. Survey of wireless 
indoor positioning techniques and systems. IEEE Transactions on Systems Man and Cybernetics Part C Applications and Reviews, 37(6):1067\{1080, 2007.

[16] Mohit Saxena, Puneet Gupta, and Bijendra Nath Jain. Experimental analysis of rssi-based location estimation in wireless sensor networks. 2008 3rd International Conference on Communication Systems Software and Middleware and Workshops COMSWARE 08, pages 503\{510, 2008.

[17] Gaddi Blumrosen, Bracha Hod, Tal Anker, Danny Dolev, and Boris Rubinsky. Continuous close-proximity rssi-based tracking in wireless sensor networks. In BSN, pages 234\{239, 2010.

[18] Elbes, M.; Al-fuqaha, A.; Rayes, A., "Gyroscope drift correction based on TDoA technology in support of Pedestrian Dead Reckoning," Globecom Workshops (GC Wkshps), 2012 IEEE , vol., no., pp.314,319, 3-7 Dec. 2012

[19] Roetenberg, D.; Luinge, H.J.; Baten, C. T M; Veltink, P.H., "Compensation of magnetic disturbances improves inertial and magnetic sensing of human body segment orientation," Neural Systems and Rehabilitation Engineering, IEEE Transactions on , vol.13, no.3, pp.395,405, Sept. 2005

[20] http://www.nursingworld.org/nursingshortage, 2014. [Online; accessed 29-December-2013]

[21] http://electroiq.com/blog/2013/04/health-awareness-spurs-quadrupling-in-mems-sensormarket-for-wea/, 2014. [Online; accessed 18-February-2014]

[22] M S Grewal, L R Weill, and A P Andrews. Global positioning systems, inertial navigation, and integration, page 364. Wiley-Interscience, 2007.

[23] Hightower, J. \& Borriello, G. (2001). Location Systems for Ubiquitous Computing, IEEE Computer, vol.34, no.8, August 2001, pp.57-66.

[24] Chuan-Chin Pu, Chuan-Hsian Pu, and Hoon-Jae Lee (2009)..

[25] Ambili Thottam Parameswaran, Mohammad Iftekhar Husain, Shambhu Upadhyaya. Is RSSI a Reliable Parameter in Sensor Localization Algorithms - An Experimental Study

[26] Pu, C.-C. (2009). Development of a New Collaborative Ranging Algorithm for RSSI Indoor Location Tracking in WSN, PhD Thesis, Dongseo University, South Korea.

[27] Savvides, A.; Han, C.-C. \& Mani, B. (2001). Strivastava. Dynamic Fine-Grained Localization in Ad-Hoc Networks of Sensors, MobiCom, pp.166-179, Rome, Italy, July 2001.

[28]Phaiboon, S. (2002). An Empirically Based Path Loss Model for Indoor Wireless Channels in Laboratory Building, IEEE TENCON, pp.1020 $\square$ 1023, vol.2, October 2002.

[29] http://tom.pycke.be/mav/70/gyroscope-to-roll-pitch-and-yaw [Online; accessed 22-July-2014]

[30] http://hyperphysics.phy-astr.gsu.edu/hbase/forces/isq.html [Online; accessed 26-July-2014]

[31] http://www.shimmersensing.com/ [Online; accessed 26-July-2014]

[32] http://www.libelium.com/ [Online; access 27-July-2014]

[33] https://www.sparkfun.com/products/11977 [Online; accessed 27-July-2014]

[34] IEEE TRANSACTIONS ON SIGNAL PROCESSING, VOL. 50, NO. 2, FEBRUARY 2002

Page 54 
A Tutorial on Particle Filters for Online Nonlinear/Non-Gaussian Bayesian Tracking, M. Sanjeev Arulampalam, Simon Maskell, Neil Gordon, and Tim Clapp

[35] N. Bergman, "Recursive Bayesian estimation: Navigation and tracking applications," Ph.D. dissertation, Linköping Univ., Linköping, Sweden, 1999.

[36] A. Doucet, "On sequential Monte Carlo methods for Bayesian filtering," Dept. Eng., Univ. Cambridge, UK, Tech. Rep., 1998.

[37] Ryder, J.; Longstaff, B.; Reddy, S.; Estrin, D. 2009 Ambulation: A tool for monitoring mobility patterns over time using mobile phones, Proceedings - 12th IEEE Int. Conf., CSE 2009, v 4, 927-931.

[38] E. Martin, O. Vinyals, G. Friedland, and R. Bajcsy, "Precise indoor localization using smart phones," in International Conference on Multimedia, ser. MM 2010, pp. 787-790.

[39] Waspmote Datasheet. http://www.libelium.com/downloads/documentation/waspmote datasheet.pdf [Online; accessed 29-December-2014]

[40] ITG-3200 Datasheet. https://www.sparkfun.com/datasheets/Sensors/Gyro/PS-ITG-3200-0001.4.pdf [Online; accessed 29-December-2014] 